\title{
Dynamics of Markov chains and stable manifolds for random diffeomorphisms
}

\author{
M. BRIN AND YU. KIFER \\ University of Maryland, College Park, MD 20742, USA; Hebrew University of \\ Jerusalem, Jerusalem, Israel
}

(Received 26 June 1986 and revised 20 October 1986)

Abstract. We consider the Markov chain on a compact manifold $M$ generated by a sequence of random diffeomorphisms, i.e. a sequence of independent $\operatorname{Diff}^{2}(M)$ valued random variables with common distribution. Random diffeomorphisms appear for instance when diffusion processes are considered as solutions of stochastic differential equations. We discuss the global dynamics of Markov chains with continuous transition densities and construct non-random stable foliations for random diffeomorphisms.

\section{Introduction}

Let $\mu$ be a probability measure on the space $\operatorname{Diff}^{2}(M)$ of $C^{2}$-diffeomorphisms of a compact Riemannian manifold $M$. By a sequence of random diffeomorphisms we mean a sequence $F_{1}, F_{2}, \ldots$ of independent $\operatorname{Diff}^{2}(M)$-valued random variables with common distribution $\mu$. Random diffeomorphisms appear for instance when diffusion processes are considered as solutions of stochastic differential equations, see $[K \mathbf{K}]$ and $[\mathbf{K i}$, chapter V]. Probability measures

$$
P(x, \Gamma)=\mu\left\{f \in \operatorname{Diff}^{2}(M): f x \in \Gamma\right\}, \quad \Gamma \subset M,
$$

give rise to a random walk $X_{n}=F_{n} X_{n-1}$. Moreover, since $F_{n}$ is a random diffeomorphism, we obtain a Markov chain $v_{n}=D F_{n} v_{n-1}$ in the tangent bundle $T M$. By the Oseledec multiplicative ergodic theorem (see Theorem 3.1 below, [Os], [Ru], [L]), if $x$ does not belong to an exceptional set, then for almost every sequence $\omega=$ $\left(f_{1}, f_{2}, \ldots\right)$ the characteristic exponent

$$
\chi(x, \omega, v)=\lim _{n \rightarrow \infty} \frac{1}{n} \ln \left\|D f_{n} \circ \ldots \circ D f_{1} v\right\|
$$

exists for every vector $v \in T_{x} M$. Moreover, there is a filtration

$$
\{0\}=V_{(x, \omega)}^{0} \subset V_{(x, \omega)}^{1} \subset \cdots \subset V_{(x, \omega)}^{\kappa}=T_{x} M
$$

such that the characteristic exponent is constant on $V_{(x, \omega)}^{i} \backslash V_{(x, \omega)}^{i-1}$. In general $\chi(x, \omega, v)$ depends non-trivially on $\omega$. However, under certain natural assumptions (see Theorem 3.3 below and [Ki, chapter III]), if $x$ does not belong to an exceptional set, then $\chi(x, \omega, v)$ almost surely does not depend on $\omega$ for all $v \in T_{x} M$ and there exists a non-random $D F_{n}$-invariant filtration of subspaces

$$
\{0\}=L_{x}^{0} \subset L_{x}^{1} \subset \cdots \subset L_{x}^{\prime}=T_{x} M
$$


such that $\chi(x, \omega, v)=\lambda_{j}(x)$ is constant on $L_{x}^{j} \backslash L_{x}^{j-1}$ with probability 1 . In the ergodic case the $\lambda_{j}$ 's do not depend on $x$.

The main purpose of this paper is to construct non-random foliations invariant under random diffeomorphisms. Denote by $F^{n}$ the composition $F_{n} \circ \cdots \circ F_{1}$. According to a version of the stable manifold theorem (see Theorem 4.1 below, $[\mathbf{R u}],[\mathbf{P}],[\mathbf{F H Y}])$, if $\chi(x, \omega, v)=\lambda<0$ for $v \in V_{(x, \omega)}^{i} \backslash V_{(x, \omega)}^{i-1}$, then the set

$$
\left\{y \in M: \limsup _{n \rightarrow \infty} \frac{1}{n} \ln \left(\operatorname{dist}\left(F^{n}(\omega) x, F^{n}(\omega) y\right)\right) \leq \lambda\right\}
$$

is a submanifold tangent to $V_{(x, \omega)}^{i}$ at $x$. The main result of this paper is Theorem 4.2 below. It states that, under certain integrability conditions, if the transition probabilities $P(x, \cdot)$ have continuous densities, then the subbundle $L^{j}$ is integrable for any $j$ with $\lambda_{j}<0$. Its integral manifolds $W_{x}^{j}$ foliate the supports of probability measures on $M$ that are invariant under the random walk $X_{n}$ and ergodic. The corresponding foliation is invariant under any $f \in \operatorname{supp}(\mu)$. Although any vector tangent to a leaf $W_{x}^{j}$ is exponentially contracted by $D F^{n}(\omega)$ with probability 1 , the entire leaf $W_{x}^{j}$ or any non-random part of it may not be contracted by $F^{n}(\omega)$ (see Example 4.7). However, for any $x$, if $\omega$ does not belong to a set of measure 0 , then there is a neighbourhood of $x$ in $W_{x}^{j}$ that is exponentially contracted by $F^{n}(\omega)$.

Note that if a non-degenerate diffusion process is represented by a sequence of random diffeomorphisms (or in this case a stochastic flow), then the transition probabilities do have continuous densities. The main results and auxiliary statements of this paper hold true if the continuity of the transitional densities is replaced by the weaker assumption that they are bounded. Moreover, it is sufficient to assume that the transition probabilities have bounded densities with respect to a continuous (non-atomic) measure. The continuity assumption allows us to simplify many proofs and to make the probabilistic arguments in $\S 2$ self-contained. In $\S 2$ we discuss the global dynamics of Markov chains with continuous transition densities. Some of the results of this section are well known for more general Markov chains, e.g. Harris or recurrent chains (see [Or], [Re, chapter 6]). In $\S 3$ we study the invariant filtrations in the tangent bundle and prove, in particular, the Hölder continuity of the non-random invariant subbundles $L^{j} . \S 4$ contains our main result, the integrability of the subbundles $L^{j}$ for which $\lambda_{j}<0$. In $\S 5$ we prove a general theorem on the Hölder continuity of invariant subbundles for partially non-uniformly hyperbolic dynamical systems.

This paper was written during the visits of the first author to the Hebrew University of Jerusalem in June 1985 and 1986 and the visits of the second author to the University of Maryland in September 1985 and 1986. Both authors were supported by BSF Israel-USA Grant \# 84-00028, and the second author was supported by NSF Grant \# DMS-82-04024 and by DARPA under NIMMP.

\section{Markov chains with continuous transition densities}

In this section we review the necessary properties of Markov chains with continuous transition densities. The arguments we give below are very similar to those usually applied in the classical case of finite Markov chains. 
Let $\left\{X_{n}\right\}$ be a Markov chain on a compact Riemannian manifold $M$ with transition probabilities $P(x, \Gamma)$, i.e. if $X_{n}=x$ then $X_{n+1} \in \Gamma$ with probability $P(x, \Gamma)$ (cf. [Re]). From now on we assume that transition probabilities have continuous transition densities, i.e. there exists a non-negative function $p(x, y)$ continuous in both variables such that for any measurable subset $\Gamma \subset M$ and any $x \in M$

$$
P(x, \Gamma)=\int_{\Gamma} p(x, y) d m(y),
$$

where $m$ is the normalized Riemannian volume in $M$. Let $P^{k}(x, \Gamma)$ be the probability of reaching $\Gamma$ from $x$ in $k$ steps and let $p^{k}(x, y)$ denote the continuous density of $P^{k}(x, \Gamma)$,

$$
P^{k}(x, \Gamma)=\int_{\Gamma} p^{k}(x, y) d m(y) .
$$

2.1. Definition. A Borel probability measure $Q$ on $M$ is called invariant under $\left\{X_{n}\right\}$, or simply, invariant if

$$
Q(\Gamma)=\int_{M} P(x, \Gamma) d Q(x)
$$

for any measurable $\Gamma \subset M$.

The existence of invariant measures for Markov chains with continuous transition densities is well known. Actually a weaker condition that the measure $P(x, \cdot)$ depends continuously on $x$ in the weak topology implies the existence of invariant measures.

2.2. LEMMA. If the family of Borel measures $P(x, \cdot)$ depends continuously on $x$ in the weak topology, then there exists at least one invariant Borel measure.

Proof. Denote by $P^{*}$ the operator in the space of measures which acts by the formula

$$
\left(P^{*} \eta\right)(\Gamma)=\int_{M} d \eta(x) P(x, \Gamma) .
$$

Let $\eta$ be an arbitrary Borel probability measure on $M$ and let

$$
\eta_{n}=\frac{1}{n} \sum_{k=0}^{n-1}\left(P^{*}\right)^{k} \eta
$$

Since the space of probability measures on a compact $M$ is compact in the weak topology (see [Ro, p. 100]), there is a subsequence $n_{i}$ such that $\eta_{n_{i}} \rightarrow^{w} \rho$ and also

$$
P^{*} \eta_{n_{i}}=\frac{1}{n_{i}} \sum_{k=1}^{n_{i}}\left(P^{*}\right)^{k} \eta \rightarrow{ }^{w} \rho .
$$

Let $P$ denote the induced action in the space of functions:

$$
(P g)(x)=\int_{M} g(y) P(x, d y) .
$$

Then for a continuous function $g$ the function $P g$ is also continuous and

$$
\int_{M} g d P^{*} \eta_{n_{i}}=\int_{M} P g d \eta_{n_{i}} \underset{i \rightarrow \infty}{\longrightarrow} \int_{M} P g d \rho=\int_{M} g d P^{*} \rho .
$$

Therefore, $P^{*} \eta_{n_{i}} \rightarrow^{w} P^{*} \rho$ and hence $P^{*} \rho=\rho$, i.e. $\rho$ is invariant. 
The continuity of $p(x, y)$ easily implies that any invariant measure $Q$ has continuous density $q(x)$. Indeed

$$
Q(\Gamma)=\int_{M} P(x, \Gamma) d Q(x)=\int_{\Gamma} d m(y) \int_{M} p(x, y) d Q(x),
$$

so that

$$
q(y)=\int_{M} p(x, y) d Q(x) .
$$

We shall call $q$ an invariant density.

2.3. Definition. A measurable subset $\Gamma \subset M$ is called invariant under $\left\{X_{n}\right\}$, or simply, invariant if

$$
P(x, \Gamma)=1
$$

for $m$-a.e. ( $m$-almost every) $x \in \Gamma$.

2.4. Definition. An invariant measure $Q$ is ergodic if for any invariant set $\Gamma$, either $Q(\Gamma)=0$ or $Q(\Gamma)=1$. We will refer to the density of $Q$ as an invariant ergodic density.

For an invariant measure $Q$ with density $q$ we write

$$
A_{q}=\{x \in M: q(x)>0\} .
$$

The open set $A_{q}$ is invariant since

$$
\begin{aligned}
0 & =Q\left(M \backslash A_{q}\right)=\int_{M \backslash A_{q}} d m(y) \int_{M} q(x) p(x, y) d m(x) \\
& =\int_{M \backslash A_{q}} d m(y) \int_{A_{q}} q(x) p(x, y) d m(x),
\end{aligned}
$$

and therefore $p(x, y)=0$ if $x \in A_{q}$ and $y \in M \backslash A_{q}$. Hence $P\left(x, A_{q}\right)=1$ when $x \in A_{q}$. Moreover, by the continuity of $p(x, y)$,

$$
P\left(x, A_{q}\right)=1 \quad \text { for any } x \in \bar{A}_{q},
$$

where $\overline{A_{q}}$ is the closure of $A_{q}$.

2.5. Proposition. Let $q$ be an invariant ergodic density. Then there exists an integer $T$ called the period of the Markov chain for which:

(i) $A_{q}$ is the union of disjoint open sets $A_{q}^{i}, 0 \leq i \leq T-1$, such that if $x \in A_{q}^{i}$ and $p(x, y)>0$, then $y \in A_{q}^{j}$, where $j=(i+1) \bmod T$, and

$$
Q\left(A_{q}^{i}\right)=\int_{A_{q}^{i}} q(x) d m(x)=\frac{1}{T} ;
$$

(ii) for every compact subset $K \subset A_{q}^{i}$ there are $N=N(K)$ and $\delta=\delta(K)>0$ such that $p^{N T}(x, y)>\delta$ for any $x \in \bar{A}_{q}^{i}$ and $y \in K$.

We shall need the following auxiliary facts.

2.6. Lemma. Let $p^{i}(x, y)>0$ and $p^{j}(y, z)>0$ for some $x, y, z \in M$ and integers $i, j$. Then $p^{i+j}(x, z)>0$. 
Proof. Recall that the functions $p^{k}(x, y)$ are continuous and that

$$
p^{i+j}(x, z)=\int_{M} p^{i}(x, v) p^{j}(v, z) d m(v),
$$

the integrand being positive in a neighborhood of $v=y$.

2.7. Lemma. For any $x \in \bar{A}_{q}$ and any $y \in A_{q}$

$$
n(x, y) \stackrel{\text { def }}{=} \inf \left\{n: p^{n}(x, y)>0\right\}
$$

is finite and the function $n(x, y)$ is upper semicontinuous in $(x, y)$.

Proof. For $x \in \bar{A}_{q}$ let

$$
U(x)=\{y: n(x, y)<\infty\} .
$$

The set $U(x)$ is clearly open, non-empty and contained in $A_{q}$. By Lemma 2.6, $U(x)$ is invariant. Hence, by the ergodicity of $q, U(x)=A_{q}$ and $n(x, y)$ is finite for any $x \in \bar{A}_{q}, y \in A_{q}$. If $p^{k}(x, y)>0$ then, by the continuity of $p, p^{k}(\tilde{x}, \tilde{y})>0$ for any $(\tilde{x}, \tilde{y})$ close enough to $(x, y)$, and hence $n(\tilde{x}, \tilde{y}) \leq n(x, y)$.

Proof of Proposition 2.5. For $x \in A_{q}$ let

$$
I(x)=\left\{i: p^{i}(x, x)>0\right\} .
$$

By Lemma 2.6, $i+j \in I(x)$ for any $i, j \in I(x)$, i.e. $I(x)$ is an additive semigroup and a simple number theory argument shows that for some big enough $i_{0}$ the elements of $I(x)$ greater than $i_{0}$ form an arithmetic progression with difference $T(x)$ equal to the greatest common divisor of $I(x)$. Let $y \in A_{q}$. By Lemma 2.7, there exist $k$ and $n$ such that $p^{k}(x, y)>0$ and $p^{n}(y, x)>0$, and hence $k+n \in I(y)$. It follows from Lemma 2.6 that $I(x) \supset k+n+I(y)$. Therefore, $T(x)$ divides $T(y)$. By the symmetry, $T(x)=T(y)$. Hence $T(x)$ is a constant which we denote by $T$.

Fix $x_{0} \in A_{q}$ and set $A_{q}^{i}=\left\{y: p^{k T+i}\left(x_{0}, y\right)>0\right.$ for some $\left.k\right\}, i=0,1, \ldots, T-1$. By Lemma 2.7, $\bigcup_{i=0}^{T-1} A_{q}^{i}=A_{q}$. Every $A_{q}^{i}$ is open since it is the union of open sets. Furthermore, the sets $A_{q}^{i}$ are disjoint. Indeed, let $y \in A_{q}^{i} \cap A_{q}^{j}$. Then $p^{k T+i}\left(x_{0}, y\right)>0$ and $p^{i T+j}\left(x_{0}, y\right)>0$ for some $k$ and $l$. By Lemma 2.7 , there is an $n$ such that $p^{n}\left(y, x_{0}\right)>0$. It follows from Lemma 2.6 that both $n+k T+i$ and $n+l T+j$ are divisible by $T$, and hence $i-j$ is divisible by $T$. Thus $i=j$. If $p^{k T+i}\left(x_{0}, x\right)>0$ and $p(x, y)>0$ then, by Lemma $2.6, p^{k T+i+1}\left(x_{0}, y\right)>0$. It follows from above that the sets $A_{q}^{i}$ are cyclically permuted by the Markov chain and so $Q\left(A_{q}^{i}\right)=1 / T$ which concludes the proof of (i).

By Lemma 2.7, for any $x, y \in A_{q}^{i}$ there is an $n$ such that $p^{n}(x, y)>0$ and, by (i), all such $n$ 's are multiples of $T$. Recall that $I\left(x_{0}\right)$ contains an arithmetic progression $\left\{T\left(i\left(x_{0}\right)+k\right)\right\}_{k=0}^{\infty}$. Fix a compact subset $K \subset A_{q}^{i}$. By Lemma 2.7, there exists $N_{0}(K)$ such that if $x \in \bar{A}_{q}^{i}$ and $y \in K$ then $p^{k}\left(x, x_{0}\right)>0$ and $p^{l}\left(x_{0}, y\right)>0$ for some $k, l<$ $N_{0}(K)$. Therefore, the set $\left\{n: p^{n}(x, y)>0\right\}$ contains an arithmetic progression $\{T(i(x, y)+k)\}_{k=0}^{\infty}$, where $i(x, y) \leq i\left(x_{0}\right)+2 N_{0}(K) / T+1$. Let $N$ be the integral part of $i\left(x_{0}\right)+2 N_{0}(K) / T+1$ and $\delta=\min _{x \in \bar{A}_{q}^{i}, y \in K} p^{N T}(x, y)$. Since $p^{N T}(x, y)$ is continuous in $(x, y)$, it follows that $\delta>0$, which proves (ii). 
As is well known, Proposition 2.5(ii) implies the following convergence of transition densities (cf. [D, p. 197]).

2.8. Proposition. Under the assumptions of Proposition 2.5, for any compact subset $K \subset A_{q}^{i}$ with $m(K)>0$, there exist $\gamma(K), 0<\gamma(K)<1$, and $C(K)>0$ such that for any $x \in \bar{A}_{q}^{i}, y \in K$ and any positive integer $k$

$$
\left|p^{k N(K) r}(x, y)-T q(y)\right| \leq C(K)(\gamma(K))^{k} .
$$

Proof. To simplify the notation set $\tau=N(K) T$. Define

$$
\bar{M}_{k}(y)=\max _{x \in \bar{A}_{q}^{i}} p^{k \tau}(x, y), \quad \underline{M}_{k}(y)=\min _{x \in \bar{A}_{q}^{i}} p^{k \tau}(x, y) .
$$

We have

$$
\begin{aligned}
\bar{M}_{k+1}(y) & =\max _{x \in \bar{A}_{q}^{i}} \int_{A_{q}^{i}} p^{\tau}(x, z) p^{k \tau}(z, y) d m(z) \\
& \leq \max _{x \in \bar{A}_{q}^{i}} \int_{A_{q}^{i}} p^{\tau}(x, z) \bar{M}_{k}(y) d m(z) \leq \bar{M}_{k}(y) .
\end{aligned}
$$

Similarly

$$
\underline{M}_{k+1}(y) \geq \underline{M}_{k}(y) .
$$

Therefore there exist the following limits

$$
\bar{M}(y)=\lim _{k \rightarrow \infty} \bar{M}_{k}(y) \geq \underline{M}(y)=\lim _{k \rightarrow \infty} \underline{M}_{k}(y) .
$$

For $x, z \in \bar{A}_{q}^{i}$ let

$$
B_{+}=\left\{v \in A_{q}^{i}: p^{\tau}(x, v)-p^{\tau}(z, v)>0\right\}
$$

and

$$
B_{-}=\left\{v \in A_{q}^{i}: p^{\tau}(x, v)-p^{\tau}(z, v)<0\right\}
$$

Since

$$
\int_{A_{q}^{i}} p^{\tau}(x, v) d m(v)=\int_{A_{q}^{i}} p^{\tau}(z, v) d m(v)=1,
$$

we have

$$
\int_{B_{+}}\left(p^{\tau}(x, v)-p^{\tau}(z, v)\right) d m(v)=-\int_{B_{-}}\left(p^{\tau}(x, v)-p^{\tau}(z, v)\right) d m(v)
$$

Thus

$$
\begin{aligned}
& p^{(k+1) \tau}(x, y)-p^{(k+1) \tau}(z, y)=\int_{A_{q}^{i}}\left(p^{\tau}(x, v)-p^{\tau}(z, v)\right) p^{k \tau}(v, y) d m(v) \\
& \leq \bar{M}_{k}(y) \int_{B_{+}}\left(p^{\tau}(x, v)-p^{\tau}(z, v)\right) d m(v) \\
&+\underline{M}_{k}(y) \int_{B_{-}}\left(p^{\tau}(x, v)-p^{\tau}(z, v)\right) d m(v) \\
&=\left(\bar{M}_{k}(y)-\underline{M}_{k}(y)\right) \int_{B_{+}}\left(p^{\tau}(x, v)-p^{\tau}(z, v)\right) d m(v) .
\end{aligned}
$$


By Proposition 2.5(ii),

$$
\int_{B_{+}} p^{\tau}(x, v) d m(v) \leq 1-\delta(K) \cdot m\left(\left(A_{q}^{i} \backslash B_{+}\right) \cap K\right)
$$

and

$$
\int_{B_{+}} p^{\top}(z, v) d m(v) \geq \delta(K) \cdot m\left(B_{+} \cap K\right) .
$$

Therefore, the last integral in (2.3) does not exceed $1-\delta(K) m(K)$. Since $x$ and $z$ were arbitrary, we see that

$$
\bar{M}_{k+1}(y)-\underline{M}_{k+1}(y) \leq(1-\delta(K) m(K))\left(\bar{M}_{k}(y)-\underline{M}_{k}(y)\right) .
$$

Hence

$$
\bar{M}(y)=\underline{M}(y)=\lim _{k \rightarrow \infty} p^{k \tau}(x, y)
$$

and

$$
\left|\bar{M}(y)-p^{k \tau}(x, y)\right| \leq C(K) \cdot(\gamma(K))^{k}
$$

where $C(K)=\max p^{\tau}(x, y)$ and $\gamma(K)=1-\delta(K) \cdot m(K)$. By the invariance of $q$ and by Proposition 2.5(i),

$$
\begin{aligned}
q(y) & =\int_{A_{q}} q(z) p^{k \tau}(z, y) d m(z)=\int_{A_{q}^{i}} q(z) p^{k z}(z, y) d m(z) \\
& \underset{k \rightarrow \infty}{\longrightarrow} \int_{A_{q}^{i}} q(z) \bar{M}(y) d m(z)=\frac{1}{T} \bar{M}(y) .
\end{aligned}
$$

2.9. THEOREM. There exist only finitely many different probability invariant ergodic measures $Q_{1}, Q_{2}, \ldots, Q_{r}$ with densities $q_{1}, q_{2}, \ldots, q_{r}$, the corresponding sets $\bar{A}_{q_{1}}, \ldots, \bar{A}_{q_{r}}$ being disjoint. For any invariant probability measure $Q$

$$
Q=\sum_{i=1}^{r} Q\left(A_{q_{i}}\right) Q_{i}
$$

We will need the following lemmas.

2.10. LemMA. For any invariant density $q$

$$
m\left(A_{q}\right) \geq\left(\max _{x, y \in M} p(x, y)\right)^{-1} .
$$

Proof. By (2.1), if $x \in A_{q}$ then

$$
\int_{A_{q}} p(x, y) d m(y)=1,
$$

and the lemma follows.

2.11. Lemma. Let $Q$ be an invariant probability measure with density $q$ and $B \subset A_{q}$ be an invariant subset with $0<Q(B)<1$.

Then there exist normalized invariant measures $Q_{1}$ and $Q_{2}$ with densities $q_{1}$ and $q_{2}$ such that $A_{q_{1}} \cap A_{q_{2}}=\varnothing, A_{q_{1}} \cup A_{q_{2}}=A_{q}$ and

$$
Q=Q\left(A_{q_{1}}\right) Q_{1}+Q\left(A_{q_{2}}\right) Q_{2} \text {. }
$$


Proof. By the invariance of $Q$ and $B$,

$$
\begin{aligned}
Q(B) & =\int_{M} d Q(x) P(x, B)=\int_{A_{q}} d Q(x) P(x, B) \\
& =\int_{B} d Q(x) P(x, B)+\int_{A_{q} \backslash B} d Q(x) P(x, B) \\
& =Q(B)+\int_{A_{q} \backslash B} d Q(x) P(x, B) .
\end{aligned}
$$

Hence $P(x, B)=0$ for $Q$-a.e. $x \in A_{q} \backslash B$, and since $Q$ and $m$ are equivalent on $A_{q}, P(x, B)=0$ for $m$-a.e. $x \in A_{q} \backslash B$. Therefore, by the invariance of $A_{q}, P\left(x, A_{q} \backslash B\right)=1$ for $m$-a.e. $x \in A_{q} \backslash B$, i.e. the set $A_{q} \backslash B$ is invariant. Now let $Q_{1}$ be the normalized restriction of $Q$ to $B$ and $Q_{2}$ be the normalized restriction of $Q$ to $A_{q} \backslash B$. It is easy to see that both measures $Q_{1}$ and $Q_{2}$ are invariant and mutually singular. Being invariant, $Q_{1}$ and $Q_{2}$ have continuous densities $q_{1}$ and $q_{2}$. The corresponding sets $A_{q_{1}}$ and $A_{q_{2}}$ obviously have the desired properties.

Proof of Theorem 2.9. Let $Q_{1}$ and $Q_{2}$ be two invariant ergodic measures with densities $q_{1}$ and $q_{2}$ and let $x \in \bar{A}_{q_{1}} \cap \bar{A}_{q_{2}}$. Then $P\left(x, A_{q_{1}} \cap A_{q_{2}}\right)=1$ by (2.1), and $A_{q_{1}} \cap A_{q_{2}}$ is an open non-empty invariant set. Since $Q_{i}\left(A_{q_{1}} \cap A_{q_{2}}\right)>0$, then, by the ergodicity, $Q_{i}\left(A_{q_{1}} \cap A_{q_{2}}\right)=1, i=1,2$. Therefore, $q_{1}=q_{2}$ by Proposition 2.8. Now the first statement of the theorem follows from Lemma 2.10. The second statement follows immediately from Lemmas 2.10 and 2.11 .

The following result implies that the union of the supports of ergodic invariant measures $A=\bigcup_{i=1}^{r} A_{q_{i}}$ (see Theorem 2.9) attracts the Markov chain $X_{n}$.

2.12. THEOREM. Let $q_{1}, \ldots, q_{r}$ be the densities of the ergodic invariant measures and $A_{q_{1}}, A_{q_{2}}, \ldots, A_{q_{r}}$ be the corresponding sets. Then

$$
N(x, \omega) \stackrel{\text { def }}{=} \inf \left\{n: X_{n}(\omega) \in \bigcup_{i=1}^{r} A_{q_{i}}=A, X_{0}(\omega)=x\right\}<\infty
$$

with probability 1. Moreover $E e^{\delta N(x, \omega)}<\infty$ for some $\delta>0$.

Proof. If $x \in A$ then $N(x, \omega)=0$. If $x \in \bar{A} \backslash A$ then $N(x, \omega)=1$. Let now $x \in M \backslash \bar{A}$ and set

$$
U(x)=\left\{y: p^{k}(x, y)>0 \text { for some } k>0\right\} .
$$

The set $U(x)$ is open and invariant. Assume that $U(x) \cap A=\varnothing$. Then $U(x) \cap \bar{A}=\varnothing$ and hence $\overline{U(x)} \cap A=\varnothing$. Since $\overline{U(x)}$ is also invariant, Lemma 2.2 implies that there exists an invariant measure supported by $\overline{U(x)}$. This contradicts Theorem 2.9. Therefore $U(x) \cap A \neq \varnothing$ and for each $x \in M$

$$
k(x) \stackrel{\text { def }}{=} \inf \left\{k: p^{k}(x, y)>0 \text { for some } y \in A\right\}<\infty .
$$

Clearly $k(x)$ is upper semicontinuous and

$$
K=\sup _{x \in M} k(x)<\infty .
$$

Since $A$ is invariant, $P^{K}(x, \bar{A})>\delta$ for some $\delta>0$ and every $x \in M$. It follows that 
$P^{K+1}(x, A)>\delta$. We have

$$
\begin{aligned}
P\{N(x, \omega)>n(K+1)\} & =P\left\{X_{n(K+1)} \in M \backslash A\right\} \\
& =\int_{M \backslash A} P^{K+1}\left(x, d z_{1}\right) \int_{M \backslash A} \cdots \int_{M \backslash A} P^{K+1}\left(x, d z_{n}\right) \\
& \leq(1-\delta)^{n} .
\end{aligned}
$$

This last inequality together with the Borel-Cantelli lemma implies the statement of the theorem.

\section{Invariant filtrations for random diffeomorphisms}

For the convenience of the reader we formulate here a version of the Oseledec multiplicative ergodic theorem for random diffeomorphisms.

Let $M$ be a compact Riemannian manifold, $F_{1}, F_{2}, \ldots$ be a sequence of independent random $C^{2}$-diffeomorphisms with a common probability distribution $\mu$ in $\operatorname{Diff}^{2}(M)$ and $X_{0}$ be a random variable independent of all $F_{n}$ with values in $M$ and distribution $Q$. Then $X_{n}=F_{n} \circ F_{n-1} \circ \cdots \circ F_{1} X_{0}$ is a Markov chain on $M$ with transition probabilities

$$
P(x, \Gamma)=\mu\left(\left\{f \in \operatorname{Diff}^{2}(M): f x \in \Gamma\right\}\right)
$$

for any $x \in M$ and any Borel $\Gamma \subset M$. Set

$$
(\Omega, \nu)=\left(\operatorname{Diff}^{2}(M)^{\mathbb{N}}, \mu^{\mathbb{N}}\right)
$$

and let $\theta$ be the shift transformation in $\Omega$

$$
\theta\left(f_{1}, f_{2}, \ldots\right)=\left(f_{2}, f_{3}, \ldots\right) .
$$

For $x \in M$ and $\omega=\left(f_{1}, f_{2}, \ldots\right) \in \Omega$ define $\tau:(M \times \Omega) \rightarrow(M \times \Omega)$ by the formula

$$
\tau(x, \omega)=\left(f_{1} x, \theta \omega\right)
$$

Suppose $Q$ is invariant under $\left\{X_{n}\right\}$ in the sense of Definition 2.1, then the measure $(Q \times \nu)$ is $\tau$-invariant. Indeed, for any measurable $A \subset M$ and $\Phi \subset \Omega$ we have

$$
\begin{aligned}
Q \times & \nu\left(\tau^{-1}(A \times \Phi)\right) \\
& =Q \times \nu\left(\left\{(x, \omega)=\left(x,\left(f_{1}, \omega^{\prime}\right)\right): f_{1} x \in A, \omega^{\prime} \in \Phi\right\}\right) \\
& =\nu(\Phi) \cdot \int_{\operatorname{Diff}^{2}(M)} Q\left(f_{1}^{-1} A\right) d \mu\left(f_{1}\right)=\nu(\Phi) \int_{M} \int_{\operatorname{Diff}^{2}(M)} 1_{f_{1}^{-1} A} d \mu\left(f_{1}\right) d Q(x) \\
& =\nu(\Phi) \int_{M} \mu\left(\left\{f_{1}: f_{1} x \in A\right\}\right) d Q(x)=\nu(\Phi) \int_{M} P(x, A) d Q(x)=\nu(\Phi) Q(A) .
\end{aligned}
$$

We shall use the following abbreviation

$$
F^{n}(\omega)=F_{n}(\omega) \circ F_{n-1}(\omega) \circ \cdots \circ F_{1}(\omega) .
$$

3.1. Theorem (see $[\mathbf{0 s}],[\mathbf{R u}],[\mathrm{Le}]$ ). Suppose that

$$
\iint \ln ^{+}\left\|D_{x} f\right\| d \mu(f) d Q(x)<\infty,
$$

where $\left\|D_{x} f\right\|$ is the norm of the differential at $x$ and $\ln ^{+} a=\max (\ln a, 0)$. Then there is a subset $\Lambda$ of full $Q \times \nu$ measure in $M \times \Omega$ such that for every $(x, \omega) \in \Lambda$ there exists 
a filtration of linear subspaces

$$
0=V_{(x, \omega)}^{0} \subset V_{(x, \omega)}^{1} \subset \cdots \subset V_{(x, \omega)}^{\kappa}=T_{x} M
$$

and a sequence of real numbers called characteristic exponents

$$
-\infty \leq \chi_{1}(x, \omega)<\chi_{2}(x, \omega)<\cdots<\chi_{\kappa}(x, \omega)<\infty
$$

with the properties

$$
\lim _{n \rightarrow \infty} \frac{1}{n} \ln \left\|D_{x} F^{n}(\omega)\right\|=\chi_{\kappa}(x, \omega)
$$

(ii) if $v \in V_{(x, \omega)}^{i} \backslash V_{(x, \omega)}^{i-1}$ then

$$
\lim _{n \rightarrow \infty} \frac{1}{n} \ln \left\|D_{x} F^{n}(\omega) v\right\|=\chi_{i}(x, \omega) ;
$$

(iii) the functions $\kappa=\kappa(x, \omega), d_{i}=d_{i}(x, \omega)=\operatorname{dim} V_{(x, \omega)}^{i}-\operatorname{dim} V_{(x, \omega)}^{i-1}$ and $\chi_{i}=$ $\chi_{i}(x, \omega), i=1,2, \ldots, \kappa$, are $\tau$-invariant;

(iv) the subspaces $V_{(x, \omega)}^{i}$ depend measurably on $(x, \omega)$ and are DF-invariant

$$
D_{x} F(\omega) V_{(x, \omega)}^{i}=V_{\tau(x, \omega)}^{i}
$$

(v) the asymptotic behaviour of the exterior power is described by the following formula:

$$
\lim _{n \rightarrow \infty} \frac{1}{n} \ln \|\left(D_{x} F^{n}(\omega)^{\wedge r} \|=\sum_{i=j+1}^{\kappa} d_{i}(x, \omega)_{\chi i}(x, \omega)+l \cdot \chi_{j}(x, \omega),\right.
$$

where $l+\sum_{i=j+1}^{\kappa} d_{i}(x, \omega)=r$ and $0<l \leq d_{j}(x, \omega)$.

3.2. Corollary (see c.f. [FHY, Proposition 1], [P, Theorem 1.1.1]). Let $(x, \omega) \in \Lambda$ and set $x_{n}=F^{n}(\omega) x, \omega_{n}=\theta^{n} \omega, \lambda=\chi_{i}(x, \omega), \mu=\chi_{i+1}(x, \omega), \lambda<\mu$. Then for any $a>0$ there is a function $C(n)=C(x, \omega, a, n) \geq 1$ such that for all $n, k \geq 0$

(i) $\left\|D_{x_{n}} F^{k}\left(\omega_{n}\right) v\right\| \leq C(n) e^{(\lambda+a) k}\|v\|$ for any $v \in V_{\left(x_{n}, \omega_{n}\right)}^{i}$;

(ii) $\left\|D_{x_{n}} F^{k}\left(\omega_{n}\right) w\right\| \geq C^{-1}(n) e^{(\mu-a) k}\|w\|$ for any $w$ from the orthogonal complement $\left(V_{\left(x_{n}, \omega_{n}\right)}^{i}\right)^{\perp}$ of $V_{\left(x_{n}, \omega_{n}\right)}^{i}$ in $T_{x_{n}} M$;

(iii) the angle between $D_{x_{n}} F^{k}\left(\omega_{n}\right) V_{\left(x_{n}, \omega_{n}\right)}^{i}$ and $D_{x_{n}} F^{k}\left(\omega_{n}\right)\left(V_{\left(x_{n}, \omega_{n}\right)}^{i}\right)^{\perp}$ is greater than $C^{-1}(n) e^{-a k}$;

(iv) the function $C(x, \omega, a, n)$ can be chosen to depend measurably on $(x, \omega, a)$ and to satisfy $C(n) \leq C(0) e^{a n}$.

The following result produces a non-random $D F$-invariant filtration.

3.3. Theorem [Ki]. Suppose $Q$ is ergodic (see Definition 2.4) and

$$
\iint\left(\ln ^{+}\left\|D_{x} f\right\|+\ln ^{+}\left\|D_{x} f^{-1}\right\|\right) d Q(x) d \mu(f)<\infty .
$$

Then for Q-a.e. $x \in M$ there exists a measurable filtration of (non-random) subspaces

$$
0=L_{x}^{0} \subset L_{x}^{1} \subset \cdots \subset L_{x}^{l}=T_{x} M
$$

and a sequence of (non-random) numbers

$$
-\infty<\lambda_{1}<\cdots<\lambda_{l}<\infty
$$


such that

$$
\lim _{n \rightarrow \infty} \frac{1}{n} \ln \left\|D_{x} F^{n}(\omega)\right\|=\lambda_{l} \quad Q \times \nu \text {-a.e.; }
$$

(ii) if $v \in L_{x}^{j} \backslash L_{x}^{j-1}$ then

$$
\lim _{n \rightarrow \infty} \frac{1}{n} \ln \left\|D_{x} F^{n}(\omega) v\right\|=\lambda_{j} \quad Q \times \nu \text {-a.e. }
$$

(iii) the subspaces $L_{x}^{j}$ depend measurably on $x$ and are DF-invariant

$$
D_{x} F(\omega) L_{x}^{j}=L_{F(\omega) x}^{j} \quad Q \times \nu \text {-a.e. }
$$

In $[\mathbf{C}]$ a similar result was proved under stronger assumptions.

The next result establishes a relation between the random filtration given by Theorem 3.1 and the non-random filtration given by Theorem 3.3. The functions $\chi_{i}$ are clearly $\tau$-invariant, and hence constant for an ergodic $Q$. Therefore, there exist $1 \leq i_{1}<i_{2}<\cdots<i_{1} \leq \kappa$ such that $\lambda_{j}=\chi_{i_{j}}(x, \omega) Q \times \nu$-a.e. The next statement follows immediately from Theorems 3.1 and 3.3.

3.4. Corollary [Ki]. (i) $L_{x}^{j} \subseteq V_{(x, \omega)}^{i_{j}} Q \times \nu$-a.e. and filtration $L$ is the maximal nonrandom subfiltration of filtration $V$ in the sense that for $Q$-a.e. $x \in M$ and any $v \in T_{x} M$

$$
\nu\left(\left\{\omega \in \Omega: v \in V_{(x, \omega)}^{i_{j+1}-1} \backslash L_{x}^{j}\right\}\right)=0
$$

(ii) Let $\left(\Omega^{m}, \nu^{m}\right)$ be the direct product of $m=\operatorname{dim} M$ copies of $(\Omega, \nu)$, then

$$
\bigcap_{n=1}^{m} V_{\left(x, \omega_{n}\right)}^{i_{j}}=L_{x}^{j}
$$

for $Q \times \nu^{m}$-almost every $\left(x, \omega_{1}, \omega_{2}, \ldots, \omega_{m}\right)$.

Let $r>0$ be the injectivity radius of $M$. If dist $(x, y)<r$, denote by $P(x, y): T_{x} M \rightarrow$ $T_{y} M$ the parallel translation from $x$ to $y$ along the unique shortest geodesic connecting $x$ and $y$. For any points $x, y \in M$ and tangent vectors $v \in T_{x} M, w \in T_{y} M$ define

$$
\operatorname{dist}(v, w)= \begin{cases}\|v\|+\|w\| & \text { if dist }(x, y) \geq r \\ \|v-P(y, x) w\| & \text { if dist }(x, y)<r .\end{cases}
$$

For $E_{x} \subset T_{x} M$ and $E_{y} \subset T_{y} M$ define

$$
\operatorname{dist}\left(E_{x}, E_{y}\right)=\max _{\substack{v \in E_{x} \\\|v\|=1}} \min _{\substack{w \in E_{y} \\\|w\|=1}} \operatorname{dist}(v, w) .
$$

If $D_{x}: T_{x} M \rightarrow T_{\tilde{x}} M$ and $D_{y}: T_{y} M \rightarrow T_{\tilde{y}} M$ are linear maps, define

$$
\operatorname{dist}\left(D_{x}, D_{y}\right)=\left\{\begin{array}{l}
\left\|D_{x}\right\|+\left\|D_{y}\right\| \quad \text { if } \max (\operatorname{dist}(x, y), \text { dist }(\tilde{x}, \tilde{y})) \geq r \\
\left\|D_{x}-P(\tilde{y}, \tilde{x}) \circ D_{y} \circ P(x, y)\right\| \quad \text { otherwise. }
\end{array}\right.
$$

3.5. Definition. Let $\Lambda \subset M$. A family $\left\{E_{x}\right\}, x \in \Lambda$, of subspaces $E_{x} \subset T_{x} M$ is called Hölder continuous in $x$ with exponent $\alpha, 0<\alpha \leq 1$, and constant $C_{h}>0$, if for any $x, y \in \Lambda$

$$
\text { dist }\left(E_{x}, E_{y}\right) \leq C_{h}(\operatorname{dist}(x, y))^{\alpha} \text {. }
$$

For a diffeomorphism $f: M \rightarrow M$ and for a number $\sigma, 0<\sigma<1$ define

$$
\|D f\|_{\sigma}=\sup _{x}\left\|D_{x} f\right\|+\sup _{x, y} \frac{\operatorname{dist}\left(D_{x} f, D_{y} f\right)}{(\operatorname{dist}(x, y))^{\sigma}} \text {. }
$$


3.6. Lemma. Let

$$
\int \ln ^{+}\|D f\|_{\sigma} d \mu(f) \stackrel{\text { def }}{=} b<\infty
$$

Then

(i) for any $\delta>0$ and $\nu$-a.e. $\omega \in \Omega$ there exists $C_{\delta}(\omega)$ such that $\left\|D F_{n}(\omega)\right\|_{\sigma} \leq$ $C_{\delta}(\omega) e^{\delta n}, n=1,2, \ldots$;

(ii) for $\nu$-a.e. $\omega \in \Omega$ there exists $\hat{C}(\omega)$ such that

$$
\prod_{i=1}^{n}\left\|D F_{i}(\omega)\right\|_{\sigma} \leq \hat{C}(\omega) e^{2 b n}, \quad n=1,2, \ldots
$$

Proof. Let $\mu_{n}=\mu\left\{f: \ln ^{+}\|D f\|_{\sigma}>\delta n\right\}$. Then

$$
\infty>\int \ln ^{+}\|D f\|_{\sigma} d \mu(f) \geq \sum_{n=0}^{\infty} \delta n\left(\mu_{n}-\mu_{n+1}\right)=\delta \sum_{n=1}^{\infty} \mu_{n}
$$

and (i) follows from the Borel-Cantelli lemma.

To prove (ii) note that, by the ergodic theorem, for $\nu$-a.e. $\omega \in \Omega$

$$
\frac{1}{n} \ln \prod_{i=1}^{n}\left\|D F_{i}(\omega)\right\|_{\sigma} \underset{n \rightarrow \infty}{\longrightarrow} \int \ln \|D f\|_{\sigma} d \mu(f) \leq b .
$$

3.7. TheOREM. Let $\mu$ satisfy (3.2) and (3.6), let $Q$ be an ergodic invariant measure with the corresponding set $A_{q}$, and let the transition probabilities $P(x, \Gamma)$ have continuous densities $p(x, y)$. Then the subbundles $L_{x}^{j}, j=1,2, \ldots, l$, from Theorem 3.3 are defined and Hölder continuous on $\bar{A}_{q}$.

Proof. By ergodicity, the characteristic exponents $\chi_{1}, \ldots, \chi_{\kappa}$ are constants on a subset $\tilde{\Lambda} \subset \Lambda$ of full $Q \times \nu$-measure in $M \times \Omega$. Fix $i<\kappa$ and set $a=\left(\chi_{i+1}-\chi_{i}\right) / 3$. Consider the following sets

$$
\begin{aligned}
\Lambda_{N} & =\{(x, \omega) \in \tilde{\Lambda}: C(x, \omega, a, 0) \leq N, \hat{C}(\omega) \leq N\}, \\
\Lambda_{N}(\omega) & =\left\{x \in M:(x, \omega) \in \Lambda_{N}\right\},
\end{aligned}
$$

where $C$ is the function introduced in Corollary 3.2. Note that $(Q \times \nu)\left(\Lambda_{N}\right) \rightarrow 1$ as $N \rightarrow \infty$.

3.8. LEMMA. For any $N>0$ there exist $C_{N}, \alpha_{N}>0$ such that

$$
\operatorname{dist}\left(V_{(x, \omega)}^{i}, V_{(y, \omega)}^{i}\right) \leq C_{N}(\operatorname{dist}(x, y))^{\alpha_{N}}
$$

for any $(x, \omega),(y, \omega) \in \Lambda_{N}$, i.e. $V_{(x, \omega)}^{i}$ is Hölder continuous in $x$ on $\Lambda_{N}$.

Proof. It follows from Corollary 3.2 and Lemma 3.6(ii) that the assumptions of Corollary 5.3 are satisfied for $\nu$-a.e. sequence of diffeomorphisms $\omega=\left\{f_{1}, f_{2}, \ldots\right\} \in$ $\Omega, f_{i} \in \operatorname{supp}(\mu)$. Therefore the subspace $V_{(x, \omega)}^{i}=E_{x}^{\lambda}$ is Hölder continuous in $x$ on the set $\Lambda_{N}(\omega)$ with exponent and constant depending only on $\chi_{i}+a=\lambda, \chi_{i+1}-a=\mu$, $a=\frac{1}{3}\left(\chi_{i+1}-\chi_{i}\right)$, and $N$.

We will now show that $L_{x}^{j}$ is Hölder continuous in $x$ on a subset of $M$ of large $Q$-measure. To do that we fix $\varepsilon>0$ and choose $N$ so that

$$
(Q \times \nu)\left(\Lambda_{N}\right) \geq 1-\varepsilon .
$$


Then

$$
\left(Q \times \nu^{m}\right)\left\{\left(x, \omega_{1}, \ldots, \omega_{m}\right):\left(x, \omega_{n}\right) \notin \Lambda_{N} \text { for some } n\right\} \leq m \varepsilon,
$$

where $m=\operatorname{dim} M$. Therefore

$$
\left(Q \times \nu^{m}\right)\left\{\left(x, \omega_{1}, \ldots, \omega_{m}\right):\left(x, \omega_{n}\right) \in \Lambda_{N}, n=1,2, \ldots, m\right\} \geq 1-m \varepsilon .
$$

Hence, by Corollary 3.4(ii) and Lemma 3.8, there is a set $\Lambda_{\varepsilon} \subset M \times \Omega^{m}$ such that

(i) $\bigcap_{n=1}^{m} V_{\left(x, \omega_{n}\right)}^{i_{i}}=L_{x}^{j}$ for any $\left(x, \omega_{1}, \ldots, \omega_{m}\right) \in \Lambda_{\varepsilon}$;

(ii) the subspaces $V_{\left(x, \omega_{n}\right)}^{i_{j}}, n=1,2, \ldots, m$, are uniformly Hölder continuous in $x$;

(iii) $\left(Q \times \nu^{m}\right)\left(\Lambda_{\varepsilon}\right) \geq 1-m \varepsilon$.

By ergodicity, $\operatorname{dim} L_{x}^{j}$ is constant $Q$-a.e., denote it by $d$. By the Fubini theorem, there is an $m$-tuple $\left(\omega_{1}^{0}, \omega_{2}^{0}, \ldots, \omega_{m}^{0}\right)$ and a subset $\Gamma_{\varepsilon} \subset M, Q\left(\Gamma_{\varepsilon}\right) \geq 1-m \varepsilon$, such that the subspaces $V_{x, n}=V_{\left(x, \omega_{n}^{0}\right)}^{i_{j}}, n=1, \ldots, m$, are uniformly Hölder continuous in $x$ on $\Gamma_{\varepsilon}$, their intersection is $L_{x}^{j}$ and $\operatorname{dim} L_{x}^{j}=d$ for any $x \in \Gamma_{\varepsilon}$.

3.9. LEMMA. For any $x \in \Gamma_{\varepsilon}$ there exists $r=r(x)>0$ such that $L^{j}$ is Hölder continuous with some constant and exponent on $\Gamma_{\varepsilon} \cap B(r, x)$, where $B(r, x) \subset M$ is the ball of radius $r$ centred at $x$.

Proof. We first use a coordinate chart at $x$ to identify a small neighbourhood $U$ of $x$ in $M$ with a small neighbourhood in $\mathbb{R}^{m}$. This allows us to identify any subspace of $T_{y} M, y \in U$, with the corresponding subspace of $T_{x} M$. Denote by $V_{y, n}^{\perp}$ the orthogonal complement of

$$
V_{y, n}=V_{\left(y, \omega_{n}^{0}\right)}^{i_{i}},
$$

$y \in \Gamma_{\varepsilon} \cap U, n=1,2, \ldots, m$. It is easy to see that the subspace $V_{y, n}^{\perp}$ depends Hölder continuously on $y \in \Gamma_{\varepsilon}$ since $V_{y, n}$ does. For every $n=1,2, \ldots, m$ fix a basis $e_{n i}(x), i=$ $1,2, \ldots, k_{n}$, of $V_{x, n}^{\perp}$. Denote by $e_{n i}(y)$ the orthogonal projection of $e_{n i}(x)$ onto $V_{y, n}^{\perp}, y \in \Gamma_{\varepsilon} \cap U$. Then the subspace $V_{y, n}$ is the space of solutions of the following linear system:

$$
\left\langle v, e_{n i}(y)\right\rangle=0, \quad i=1, \ldots, k_{n},
$$

where $\langle$,$\rangle denotes the standard inner product in \mathbb{R}^{m}$. Recall that $L_{y}^{j}=\bigcap_{n=1}^{m} V_{y, n}$ and hence the subspace $L_{y}^{j}$ is the set of solutions of the system of linear equations

$$
\left\langle v, e_{n i}(y)\right\rangle=0, \quad i=1, \ldots, k_{n}, n=1, \ldots, m .
$$

The coefficients of this system are Hölder continuous functions of $y \in \Gamma_{\varepsilon} \cap U$. Since $\operatorname{dim} L_{y}^{j}=d$, the dimension of the space of solutions of (3.7) is $d$. In particular, there is a subset of $m-d$ equations which is equivalent to the whole system (3.7) at $x$ and a non-zero $(m-d)$-minor of that subsystem at $x$. If $r(x)$ is small enough, the corresponding minor is uniformly bounded away from 0 for $y \in B(r, x) \cap \Gamma_{\varepsilon}$ and the corresponding subsystem is equivalent to (3.7). Any scheme of solving $m-d$ linear equations applied consistently to different $y \in B(r, x) \cap \Gamma_{\varepsilon}$ will produce a basis of $L_{y}^{j}$ depending Hölder continuously on $y$. This implies the Hölder continuity of $L^{j}$ on $B(r, x) \cap \Gamma_{\varepsilon}$. 
Let $\Gamma \subset \Gamma_{\varepsilon}$ be a closed subset with

$$
Q(\Gamma) \geq 1-2 m \varepsilon .
$$

Cover $\Gamma$ by finitely many balls $B\left(r\left(x_{i}\right) / 2, x_{i}\right), i=1,2, \ldots, N_{1}$, where $r\left(x_{i}\right)$ are from Lemma 3.9. By Lemma 3.9, the subspace $L_{x}^{j}$ is Hölder continuous on each intersection $\Gamma_{E} \cap B\left(r\left(x_{i}\right), x_{i}\right)$. Let $C_{1}$ be the worst constant and $\alpha$ be the worst exponent. If $x, y \in \Gamma$ and dist $(x, y)<\frac{1}{2} \min _{i} r\left(x_{i}\right)$, then $x$ and $y$ lie in one of the balls, and therefore

$$
\operatorname{dist}\left(L_{x}^{j}, L_{y}^{j}\right) \leq C_{1}(\operatorname{dist}(x, y))^{\alpha} .
$$

For a bigger constant $C_{2}$ we have

$$
\operatorname{dist}\left(L_{x}^{j}, L_{y}^{j}\right) \leq C_{2}(\operatorname{dist}(x, y))^{\alpha} \quad \text { for any } x, y \in \Gamma \text {. }
$$

We will now use the invariance of $L^{j}$ under the random diffeomorphisms to show that $L^{j}$ is Hölder continuous on $\overline{A_{q}}$. Since the invariant density $q$ is positive on $A_{q}$ and since $Q(\Gamma) \geq 1-2 m \varepsilon$, we can choose $\varepsilon$ so small that the volume of $\Gamma$ is big enough:

$$
m(\Gamma)>m\left(A_{q}\right)\left(1-\left(3 \bar{p} m\left(A_{q}\right)\right)^{-1}\right),
$$

where $\bar{p}=\max p(x, y)$. Then for any $x \in \bar{A}_{q}$

$$
P\left(x, A_{q} \backslash \Gamma\right)=\int_{A_{q} \backslash \Gamma} p(x, y) d m(y) \leq \bar{p} \cdot m\left(A_{q} \backslash \Gamma\right)<\frac{1}{3} .
$$

On the other hand, by $(2.1), P\left(x, A_{q}\right)=1$ and therefore $P(x, \Gamma)>\frac{2}{3}$. Denote by $\mathscr{F}(C)$ the set of diffeomorphisms $f$ such that $\sup _{x}\left\|D_{x} f\right\| \leq C$ and for any $z, w \in M$ and any subspaces $S_{z} \subset T_{z} M, S_{w} \subset T_{w} M$

$$
\operatorname{dist}\left(D_{z} f^{-1} S_{z}, D_{w} f^{-1} S_{w}\right) \leq C\left(\operatorname{dist}\left(S_{z}, S_{w}\right)+\operatorname{dist}(z, w)\right) .
$$

Choose $C_{3}$ so that $\mu\left(\mathscr{F}\left(C_{3}\right)\right)>\frac{2}{3}$. Then for any $x, y \in \bar{A}_{q}$ there is a diffeomorphism $f_{x y}=f \in \mathscr{F}\left(C_{3}\right)$ such that $f x, f y \in \Gamma$. Therefore if $L_{x}^{j}$ and $L_{y}^{j}$ are defined, then for $f=f_{x y}$ we have

$$
\text { dist } \begin{aligned}
\left(L_{x}^{j}, L_{y}^{j}\right) & \leq C_{3}\left(\operatorname{dist}\left(L_{f x}^{j}, L_{f y}^{j}\right)+\operatorname{dist}(f x, f y)\right) \\
& \leq C_{3}\left(C_{2}+1\right)(\operatorname{dist}(f x, f y))^{\alpha} \leq C_{3}\left(C_{2}+1\right) C_{3}^{\alpha}(\operatorname{dist}(x, y))^{\alpha},
\end{aligned}
$$

Hence the distribution $L^{j}$ is Hölder continuous in $x$ with constant $C_{h}=C_{3}^{1+\alpha}\left(C_{2}+1\right)$ and exponent $\alpha$ on the set of full $Q$-measure in $\bar{A}_{q}$ where it is defined. By the uniform continuity, $L_{x}^{j}$ can be defined for any $x \in \bar{A}_{q}$ so that $L^{j}$ is Hölder continuous and invariant under any $f \in \operatorname{supp}(\mu)$.

We extend by continuity the subbundles $L^{j}, 1 \leq j \leq l$, to the set $\overline{A_{q}}$ and in what follows use the same notations for the extended subbundles. Since $L$ is $D F$-invariant, the statement of Corollary 3.4 holds for any $x \in \bar{A}_{q}$.

3.10. Corollary. Using the notations of Corollary 3.4, if $x \in \bar{A}_{q}$ then $L_{x}^{j} \subseteq V_{(x, \omega)}^{i_{j}}$ $\nu$-a.e. and there exists a subset $\Omega_{x}^{m}$ of full $\nu^{m}$-measure in $\Omega^{m}$ such that $\bigcap_{n=1}^{m} V_{\left(x, \omega_{n}\right)}^{i_{j}}=L_{x}^{j}$ for any $\left(\omega_{1}, \omega_{2}, \ldots, \omega_{m}\right) \in \Omega_{x}^{m}$.

\section{Invariant foliations for random diffeomorphisms}

In this section we prove that if $\lambda_{j}=\chi_{i_{j}}<0$, then the subbundle $L^{j}$ is integrable, see Theorem 4.2 below. Its integral manifolds $W_{x}^{j}$ are contracted in a certain probabilistic 
sense and form a foliation $W^{j}$ invariant under any diffeomorphism from the support of $\mu$. Throughout this section we assume that $M$ is compact.

The version of the stable manifold theorem for a sequence of random diffeomorphisms $F_{1}, F_{2}, \ldots, F_{n}, \ldots$ formulated below follows immediately from Theorem 5.1 in [Ru] and Theorem 5.1 in [Ru 1]. We keep the notations of Theorem 3.1 introduced at the beginning of $\S 3$.

4.1. The Stable manifold theOrem (see also [P], [FHY]). Let (3.6) be satisfied and let $(x, \omega) \in \Lambda$ and $\xi<0$ satisfy $\chi_{i}(x, \omega)<\xi<\chi_{i+1}(x, \omega)$. Then there exist $\delta=$ $\delta(x, \omega)>0$ and $R=R(x, \omega, \xi)>0$ such that

(i) $W_{(x, \omega)}^{\xi}=\left\{y\right.$ : dist $(x, y)<\delta$ and $\operatorname{dist}\left(F^{n}(\omega) x, F^{n}(\omega) y\right) \leq R e^{\xi n} \operatorname{dist}(x, y)$ for all $n \geq 0\}$ is a $C^{1}$-submanifold tangent to $V_{(x, \omega)}^{i}$ at $x$ and diffeomorphic with the unit ball in $V_{(x, \omega)}^{i}$;

(ii) $(y, \omega) \in \Lambda$ for any $y \in W_{(x, \omega)}^{\xi}$ and the tangent space $T_{y} W_{(x, \omega)}^{\xi}$ is $V_{(y, \omega)}^{i}$;

(iii) for any $y, z \in W_{(x, \omega)}^{\xi}$, dist $\left(F^{n}(\omega) y, F^{n}(\omega) z\right) \leq R e^{\xi n} \operatorname{dist}(y, z)$ for all $n \geq 0$;

(iv) the functions $R^{-1}\left(F^{n}(\omega) x, \theta^{n} \omega, \xi\right)$ and $\delta\left(F^{n}(\omega) x, \theta^{n} \omega\right)$ may decrease only subexponentially in $n$;

(v) denote by $W$ the connected component of $F_{1}(\omega) x$ in the intersection of $F_{1}(\omega) W_{(x, \omega)}^{\xi}$ with the ball of radius $\delta\left(F_{1}(\omega) x, \theta \omega\right)$ centred at $F_{1}(\omega) x$; then $W \subset$ $W_{\left(F_{1}(\omega) x, \theta \omega\right)}^{\xi}$.

The main result of this section is the following theorem.

4.2. THEOREM. Let $\mu$ satisfy (3.2) and (3.6), let $Q$ be an ergodic invariant measure with the corresponding set $A_{q}$, and let the transition probabilities $P(x, \Gamma)$ have continuous densities $p(x, y)$. Suppose $\lambda_{j}=\chi_{i_{j}}<0$.

Then the subbundle $\left\{L_{x}^{j}\right\}$ is integrable in the following sense. There exists a foliation $W^{j}$ of $A_{q}$ into $C^{1}$ complete submanifolds $W_{x}^{j}$ without boundary such that the tangent space $T_{x} W_{x}^{j}$ is $L_{x}^{j}$ for any point $x \in A_{q}$. The foliation $W^{j}$ is invariant under any diffeomorphism $f$ from the support of $\mu$. For any $x \in A_{q}$ and $\nu$-almost every $\omega$ there exists a neighbourhood $W_{x, \varepsilon(\omega)}^{j}$ of $x$ in $W_{x}^{j}$ such that

$$
\limsup _{n \rightarrow \infty} \frac{1}{n} \ln \operatorname{diam}\left(F^{n}(\omega) W_{x, \varepsilon(\omega)}^{j}\right) \leq \lambda_{j} .
$$

Proof. We first give an outline of the argument. For an $x \in A_{q}$ and a typical sequence $\omega$ of diffeomorphisms $f_{n} \in \operatorname{supp}(\mu)$ we will construct a special sequence of norms \|\|$\quad \|_{n}$. The non-uniformly hyperbolic sequence of matrices $D_{x_{n}} f_{n+1}, x_{n}=f_{n} x_{n-1}, x_{0}=$ $x$, will be uniformly hyperbolic if the norms \|\|$_{n}$ are used. Then, after establishing certain properties of the norms \|\|$\|_{n}$, we will use them to prove that any piecewise smooth curve, tangent to $L^{j}$ and passing through $x$, is exponentially contracted by the sequence of diffeomorphisms $\left\{f_{n}\right\}$, see Lemma 4.5 below. This geometric property will imply the integrability of $L^{j}$.

Fix $x \in A_{q}$ and $\omega \in \Omega=\left(\operatorname{Diff}^{2}(M)\right)^{\mathbb{N}}, \omega=\left\{f_{n}\right\}$, such that $L_{x}^{j} \subseteq V_{(x, \omega)}^{i_{j}}$, cf. Corollary $3.10,(x, \omega) \in \Lambda$ (see Theorem 3.1), and the numbers $\chi_{i_{j}}(x, \omega)<\chi_{i_{j}+1}(x, \omega)$ are typical values (recall that, by ergodicity, the exponents are constant $Q \times \nu$-a.e.). Let $\varphi_{n}: \mathcal{N} \rightarrow$ $M$ be the exponential map at $x_{n}=f^{n} x, f^{n}=f_{n} \circ \cdots \circ f_{1}$, restricted to a neighbourhood 
$\mathcal{N} \subset \mathbb{R}^{m}=T_{x_{n}} M$ of the origin, $m=\operatorname{dim} M$. By the compactness of $M$, we may choose $\mathcal{N}$ so small that all $\varphi_{n}$ 's are one-to-one with uniformly bounded first and second derivatives of $\varphi_{n}$ and $\varphi_{n}^{-1}$. Set $\tilde{f}_{n}=\varphi_{n}^{-1} \circ f_{n} \circ \varphi_{n-1}: \mathcal{N} \rightarrow \mathbb{R}^{m}$ and introduce the following notations:

$$
\begin{gathered}
T_{n}=D_{0} \tilde{f}_{n+1}, \quad E_{0}^{s}=D \varphi_{0}^{-1}\left(V_{(x, \omega)}^{i_{j}}\right), \quad E_{0}^{u}=\left(E_{0}^{s}\right)^{\perp}, \\
E_{n+1}^{s}=T_{n} E_{n}^{s}=D \varphi_{n+1}^{-1}\left(V_{\left(f^{n+1} x_{0} \theta^{n+1} \omega\right)}^{i_{j}}\right), \quad E_{n+1}^{u}=T_{n} E_{n}^{u}, \\
T_{n}^{k}=T_{n+k-1} \cdots T_{n}, \quad S_{n}^{k}=T_{n}^{k}\left|E_{n}^{s}, \quad U_{n}^{k}=T_{n}^{k}\right| E_{n}^{u},
\end{gathered}
$$

where $n, k \geq 0, T_{n}^{0}=\mathrm{id}, S_{n}^{0}=\mathrm{id}, U_{n}^{0}=\mathrm{id}$. Set

and fix $a>0$ such that

$$
\lambda=\chi_{i_{j}}, \quad \mu=\chi_{i_{j}+1}
$$

$$
a<\min \left(\frac{-\alpha \lambda}{2 \alpha+5}, \frac{\mu-\lambda}{10}\right)
$$

where $\alpha$ is the Hölder exponent of $L^{j}$. By Corollary 3.2 there is a function $C(n)$ such that for all $n, k \geq 0$

$$
\begin{aligned}
\left\|S_{n}^{k} v^{s}\right\| & \leq C(n) e^{(\lambda+a) k}\left\|v^{s}\right\|, \quad v^{s} \in E_{n}^{s}, \\
\left\|U_{n}^{k} v^{u}\right\| & \leq C^{-1}(n) e^{(\mu-a) k}\left\|v^{u}\right\|, \quad v^{u} \in E_{n}^{u}, \\
\left\|v^{s}\right\| & \leq C(n)\|v\|, \quad\left\|v^{u}\right\| \leq C(n)\|v\|,
\end{aligned}
$$

where $v=v^{s}+v^{u}, v^{s} \in E_{n}^{s}, v^{u} \in E_{n}^{u}$,

$$
1 \leq C(n) \leq C(0) e^{a n} .
$$

The following sequence of norms is usually called the Lyapunov metric or the metric adjusted to the dynamical system:

$$
\begin{aligned}
& \left\|v^{s}\right\|_{n}=\sum_{k=0}^{\infty} e^{-(\lambda+2 a) k}\left\|S_{n}^{k} v^{s}\right\|, \quad v^{s} \in E_{n}^{s}, \quad n \geq 0, \\
& \left\|v^{u}\right\|_{n}=\sum_{k=0}^{n} e^{(\mu-2 a) k}\left\|\left(U_{n-k}^{k}\right)^{-1} v^{u}\right\|, \quad v^{u} \in E_{n}^{u}, \quad n \geq 0, \\
& \|v\|_{n}^{2}=\left\|v^{s}\right\|_{n}^{2}+\left\|v^{u}\right\|_{n}^{2} \quad \text { for } v=v^{s}+v^{u}, \quad v^{s} \in E_{n}^{s}, \quad v^{u} \in E_{n}^{u} .
\end{aligned}
$$

4.3. LEMMA [BN]. The sequence of norms $\left\{\|\| \|_{n}\right\}$ satisfies

(i) i\| $S_{n} v^{s}\left\|_{n+1} \leq e^{\lambda+2 a}\right\| v^{s} \|_{n}, \quad v^{s} \in E_{n}^{s}$,

(ii) $\left\|U_{n} v^{u}\right\|_{n+1} \geq e^{\mu-2 a}\left\|v^{u}\right\|_{n}, \quad v^{u} \in E_{n}^{u}$,

(iii) $\frac{1}{2}\|v\| \leq\|v\|_{n} \leq C_{1} e^{2 a n}\|v\|$, where $C_{1}=2 C^{2}(0) /\left(1-e^{-a}\right)$.

Proof. We have

$\left\|S_{n} v^{s}\right\|_{n+1}=\sum_{k=0}^{\infty} e^{-(\mu+2 a) k}\left\|S_{n+1}^{k} S_{n} v^{s}\right\| \leq e^{\lambda+2 a} \sum_{k=0}^{\infty} e^{-(\lambda+2 a)(k+1)}\left\|S_{n}^{k+1} v^{s}\right\| \leq e^{\lambda+2 a}\|v\| \|_{n}$, and hence (i) holds. Similarly

$$
\begin{aligned}
\left\|U_{x} v^{u}\right\|_{n+1} & =\sum_{k=0}^{n+1} e^{(\mu-2 a) k}\left\|\left(U_{n+1-k}^{k}\right)^{-1} U_{n} v^{u}\right\| \\
& =\left\|U_{n} v^{u}\right\|+e^{\mu-2 a} \sum_{k=1}^{n+1} e^{(\mu-2 a)(k-1)}\left\|\left(U_{n+1-k}^{k-1}\right)^{-1} v^{u}\right\| \\
& \geq e^{-2 a}\left\|v^{u}\right\|_{n},
\end{aligned}
$$

and hence (ii) holds. 
To prove (iii) we first note that the zero terms in the right-hand sides of (4.6) and (4.7) are $\left\|v^{s}\right\|$ and $\left\|v^{u}\right\|$. Therefore $\left\|v^{s}\right\|\left\|_{n} \geq\right\| v^{s} \|$ and $\left\|v^{u}\right\|_{n} \geq\left\|v^{u}\right\|$. This implies the left inequality in (iii) since

$$
\|v\| \leq\left\|v^{s}\right\|+\left\|v^{u}\right\| \leq 2 \max \left(\left\|v^{s} \mid\right\|_{n},\left\|v^{u}\right\|_{n}\right) .
$$

To prove the right inequality observe that, by (4.2) and (4.5),

$$
\left\|v^{s}\right\|_{n} \leq \sum_{k=0}^{\infty} e^{-(\lambda+2 a) k} C(n) e^{(\lambda+a) k}\left\|v^{s}\right\| \leq \frac{C(0)}{1-e^{-a}} e^{a n}\left\|v^{u}\right\| .
$$

Similarly, by (4.3) and (4.5),

$$
\left\|v^{u}\right\|_{n} \leq \sum_{k=0}^{n} e^{(\mu-2 a) k} C(n-k) e^{-(\mu-a) k}\left\|v^{u}\right\| \leq \frac{C(0)}{1-e^{-2 a}} e^{a n}\left\|v^{u}\right\| .
$$

By the last two estimates, (4.6) and (4.7),

$$
\begin{aligned}
\|v\|_{n}^{2} & \leq C^{2}(0) e^{2 a n}\left(\frac{\left\|v^{s}\right\|^{2}}{\left(1-e^{-a}\right)^{2}}+\frac{\left\|v^{u}\right\|^{2}}{\left(1-e^{-2 a}\right)^{2}}\right) \\
& \leq 2 C^{2}(0) e^{2 a n} \frac{C^{2}(n)}{\left(1-e^{-a}\right)^{2}}\|v\|^{2} \leq 2 \frac{C^{4}(0)}{\left(1-e^{-a}\right)^{2}} e^{4 a n}\|v\|^{2} .
\end{aligned}
$$

Denote by dist ${ }_{n}$ and length $h_{n}$ the distance in $\mathbb{R}^{m}$ and the length of curves induced by the norm \|\|$\quad\|\|_{n}$. Then, by Lemma 4.3(iii)

$$
\frac{1}{2} \operatorname{dist}(y, z) \leq \operatorname{dist}_{n}(y, z) \leq C_{1} e^{2 a n} \operatorname{dist}(y, z)
$$

for any points $y, z \in \mathbb{R}^{m}$, and

$$
\frac{1}{2} \text { length }(\sigma) \leq \text { length }_{n}(\sigma) \leq C_{1} e^{2 a n} \text { length }(\sigma)
$$

for any piecewise smooth curve $\sigma$. For any matrix $T$ and any vector $v$ we have, by Lemma 4.3(iii)

$$
\|T v\|_{n+1} \leq C_{1} e^{2 a(n+1)}\|T v\| \leq C_{1} e^{2 a(n+1)}\|T\| \cdot\|v\| \leq 2 C_{1} e^{2 a(n+1)}\|T\| \cdot\|v\|_{n}
$$

and hence

$$
\|T v\|_{n+1} \leq 2 C_{1} e^{2 a(n+1)}\|T\| \cdot\|v\|_{n} .
$$

Let $y \in \mathcal{N}$ be such that $\varphi_{n}(y) \in A_{q}$. Set

$$
\tilde{L}_{n}^{j}(y)=\left(D \varphi_{n}\right)^{-1}\left(L_{\varphi_{n}(y)}^{j}\right) .
$$

Since $L^{j}$ is Hölder continuous with exponent $\alpha$ and constant $C_{h}$, the distributions $\tilde{L}_{n}^{j}$ are uniformly Hölder continuous with the same exponent $\alpha$ and constant $\tilde{C}_{h}$.

4.4. LemmA. Let $\varphi_{n}(y) \in A_{q}$ and let $v \in \tilde{L}_{n}^{j}(y)$ and denote by $v^{s}$ and $v^{u}$ the components of $v$ that are parallel to $E_{n}^{s}$ and $E_{n}^{u}$ respectively, $v=v^{s}+v^{u}$. Then

$$
\frac{\left\|v^{u}\right\|_{n}}{\|v\|_{n}} \leq C_{4} e^{3 a n}\left(\operatorname{dist}_{n}(y, 0)\right)^{\alpha}
$$

for some constant $C_{4}$.

Proof. Denote by $v^{\perp}$ the component of $v$ perpendicular to $E_{n}^{s}$. Since $\tilde{L}_{n}^{j}(0) \subset E_{n}^{s}$ and $\tilde{L}_{n}^{j}$ is Hölder continuous, by (4.9)

$$
\left\|v^{\perp}\right\| \leq C_{2}(\operatorname{dist}(y, 0))^{\alpha}\|v\| \leq 2 C_{2}\left(\operatorname{dist}_{n}(y, 0)\right)^{\alpha}\|v\|
$$


for a constant $C_{2}$. Therefore by (4.4) and (4.5)

$$
\left\|v^{u}\right\| \leq C(n)\left\|v^{\perp}\right\| \leq 2 C(0) C_{2} e^{a n}\left(\operatorname{dist}_{n}(y, 0)\right)^{\alpha}\|v\| .
$$

Hence, by Lemma 4.3(iii),

$$
\frac{\left\|v^{u}\right\|_{n}}{\|v\|_{n}} \leq \frac{C_{1} e^{2 a n}\left\|v^{u}\right\|}{\frac{1}{2}\|v\|} \leq 4 C(0) C_{2} e^{3 a n}\left(\operatorname{dist}_{n}(y, 0)\right)^{\alpha} .
$$

Denote by $\tilde{f}^{n}$ the composition $\tilde{f}_{n} \circ \tilde{f}_{n-1} \circ \cdots \circ \tilde{f}_{1}$.

4.5. Lemma. Let $\omega$ in addition satisfy Lemma 3.6(i) and let $\beta$ be such that

$$
\lambda+2 a<\beta<\min (-6 a / \alpha,-3 a / \sigma),
$$

where $\alpha$ is the Hölder exponent of $L^{j}$ and a satisfies (4.1). Then there exists $\varepsilon>0$ such that for any piecewise smooth curve $\gamma:[0,1] \rightarrow \mathcal{N}$ with $\gamma(0)=0$, length $(\gamma) \leq \varepsilon$ and $\dot{\gamma}(t) \in \tilde{L}_{0}^{j}(\gamma(t)), t \in[0,1]$, the following holds:

(i) $\tilde{f}^{n}(\gamma(t)) \in \mathcal{N}$ for $t \in[0,1], \quad n \geq 0$,

(ii) length ${ }_{n}\left(\tilde{f}^{n} \circ \gamma\right) \leq e^{\beta n}$ length $_{0}(\gamma), \quad n \geq 0$,

(iii) length $\left(\tilde{f}^{n} \circ \gamma\right) \leq 2 C_{1} e^{\beta n}$ length $(\gamma), \quad n \geq 0$.

Proof. We first prove (i) and (ii) by induction in $n$. For $n=0$, (i) and (ii) are obviously true. Suppose (i) and (ii) hold for some positive integer $n$. Set $\gamma_{n}(t)=$ $\tilde{f}^{n}(\gamma(t)), t \in[0,1]$, and denote by $T_{n}(t)$ the differential $D_{\gamma_{n}}(t) \tilde{f}_{n+1}$. Let $C_{4}$ be an upper bound for the first and second derivatives of $\tilde{f}_{n}$. We have for any $t \in[0,1]$

$$
\left\|T_{n}(t) \dot{\gamma}_{n}(t)\right\|_{n+1} \leq\left\|\left(T_{n}(t)-T_{n}\right) \dot{\gamma}_{n}(t)\right\|_{n+1}+\left\|T_{n} \dot{\gamma}_{n}(t)\right\|_{n+1} .
$$

By (4.11) and (4.10)

$$
\begin{aligned}
& \left\|\left(T_{n}(t)-T_{n}\right) \dot{\gamma}_{n}(t)\right\|_{n+1} \\
& \quad \leq 2 C_{1} e^{2 a(n+1)} \cdot\left\|T_{n}(t)-T_{n}\right\| \cdot\left\|\dot{\gamma}_{n}(t)\right\|_{n} \\
& \quad \leq 2 C_{1} e^{2 a(n+1)}\left\|D \tilde{f}_{n+1}\right\|_{\sigma}\left(2 \text { length }_{n}\left(\dot{\gamma}_{n}\right)\right)^{\sigma} \cdot\left\|\dot{\gamma}_{n}(t)\right\|_{n} .
\end{aligned}
$$

Denote by $\dot{\gamma}_{n}^{s}(t)$ and $\dot{\gamma}_{n}^{u}(t)$ the components of $\dot{\gamma}_{n}(t)$ parallel to $E_{n}^{s}$ and $E_{n}^{u}$ respectively. Then

$$
\left\|T_{n} \dot{\gamma}_{n}(t)\right\|\left\|_{n+1} \leq\right\| T_{n} \dot{\gamma}_{n}^{s}(t)\left\|_{n+1}+\right\| T_{n} \dot{\gamma}_{n}^{u}(t) \|_{n+1} .
$$

It follows from (4.11) and Lemma 4.4 that

$$
\begin{aligned}
\left\|T_{n} \dot{\gamma}_{n}^{u}(t)\right\| & \leq 2 C_{1} e^{2 a(n+1)}\left\|T_{n}\right\| \cdot\left\|\dot{\gamma}_{n}^{u}(t)\right\|_{n} \\
& \leq 2 C_{1} e^{2 a(n+1)}\left\|D \tilde{f}_{n+1}\right\| \sigma\left\|\dot{\gamma}_{n}^{u}(t)\right\|_{n} \\
& \leq 2 C_{1} e^{2 a(n+1)} C_{4} e^{3 a n}\left\|D \tilde{f}_{n+1}\right\|_{\sigma}\left(\text { length }_{n}\left(\gamma_{n}\right)\right)^{\alpha}\left\|\dot{\gamma}_{n}(t)\right\|_{n} .
\end{aligned}
$$

By Lemma 4.3(i),

$$
\left\|T_{n} \dot{\gamma}_{n}^{s}(t)\right\|_{n+1} \leq e^{\lambda+2 a}\left\|\dot{\gamma}_{n}^{s}(t)\right\|_{n} \leq e^{\lambda+2 a}\left\|\dot{\gamma}_{n}(t)\right\|_{n} .
$$

Set $\delta=a$ in Lemma 3.6(i). We now put together (4.13)-(4.16) and use the inductive assumption to obtain

$$
\begin{aligned}
\left\|T_{n}(t) \dot{\gamma}_{n}(t)\right\|_{n+1} \leq & {\left[C _ { 5 } \| D \tilde { f } _ { n + 1 } \| _ { \sigma } \left(\exp ((2 a+\sigma \beta) n) \cdot \varepsilon^{\sigma}\right.\right.} \\
& \left.\left.+\exp ((5 a+\alpha \beta) n) \varepsilon^{\alpha}\right)+e^{\lambda+2 a}\right] \cdot\left\|\dot{\gamma}_{n}(t)\right\|_{n} \\
\leq & {\left[C _ { 5 } C _ { a } ( \omega ) \left(\exp ((3 a+\sigma \beta) n) \varepsilon^{\sigma}\right.\right.} \\
& \left.\left.+\exp ((6 a+\alpha \beta) n) \varepsilon^{\alpha}\right)+e^{\lambda+2 a}\right] \cdot\left\|\dot{\gamma}_{n}(t)\right\|_{n},
\end{aligned}
$$


where $C_{5}$ is a constant and $C_{a}(\omega)$ is from Lemma 3.6. By our choice of $a$ and $\beta$ (cf. (4.1) and (4.12)), it follows from the last estimate that for small enough $\varepsilon>0$

$$
\left\|T_{n}(t) \dot{\gamma}_{n}(t)\right\|\left\|_{n+1} \leq e^{\beta}\right\| \dot{\gamma}_{n}(t) \|_{n} .
$$

This implies (ii) with $n$ replaced by $n+1$ since

$$
\text { length }_{n+1}\left(\gamma_{n+1}\right)=\int_{0}^{1}\left\|T_{n}(t) \dot{\gamma}_{n}(t)\right\|_{n+1} d t .
$$

Statement (i) follows from (ii) if $\varepsilon$ is less than half the radius of the biggest ball centred at the origin and contained in $\mathcal{N}$. Statement (iii) follows immediately from (ii) and (4.10).

We are ready now to prove the integrability of $L^{j}$. Let $x \in A_{q}$. Then, by Corollary 3.10 , there is a subset $\Omega_{x}^{m}$ of full $\nu^{m}$-measure in $\Omega^{m}$ such that

$$
\bigcap_{n=1}^{m} V_{\left(x, \omega_{n}\right)}^{i_{i}}=L_{x}^{j} \quad \text { for any }\left(\omega_{1}, \omega_{2}, \ldots, \omega_{m}\right) \in \Omega_{x}^{m}
$$

Since $Q$ is ergodic we may assume that the characteristics exponents are constant for all $\omega_{i}$ 's. Fix such a collection $\left(\omega_{1}, \ldots, \omega_{m}\right)$ and a number $a>0$ satisfying (4.1) with $\lambda=\chi_{i_{j}}, \mu=\chi_{i_{j}}+1$. Choose $\beta<\lambda+3 a$ satisfying (4.12) and set $\xi=\lambda+4 a<\mu$. By Theorem 4.1, there are local stable manifolds $W_{\left(x, \omega_{n}\right)}^{\xi}, n=1, \ldots, m$. Denote by $W_{x, \varepsilon}^{j}$ the set of points lying on piecewise $C^{1}$-curves $\gamma:[0,1] \rightarrow M$ such that $\gamma(0)=$ $x, \dot{\gamma}(t) \in L_{\gamma(t)}^{j}, 0 \leq t \leq 1$, and length $(\gamma) \leq \varepsilon$. If $\varepsilon$ is small enough, we can lift such a curve $\gamma$ to the neighbourhood $\mathcal{N} \subset \mathbb{R}^{m}=T_{x} M$, apply Lemma 4.5 and obtain that

$$
\text { length }\left(F^{n}(\omega) \circ \gamma\right) \leq 2 C_{1} C_{6}^{2} e^{\beta n} \text { length }(\gamma) \text {, }
$$

where $C_{6}$ is an upper bound for the norm of the differential of the exponential map restricted to $\mathcal{N}$ and for the inverse. Assume now that $\varepsilon$ is less than the sizes $\delta\left(x, \omega_{n}\right)$ of the local stable manifolds $W_{\left(x, \omega_{n}\right)}^{\xi}, n=1,2, \ldots, m$. Then by Theorem 4.1 and (4.18)

$$
W_{x, \varepsilon}^{j} \subset \bigcap_{n=1}^{m} W_{\left(x, \omega_{n}\right)}^{\xi} \stackrel{\text { def }}{=} W .
$$

We use a coordinate chart at $x$ to identify a small neighbourhood of the origin in $\mathbb{R}^{m}$. Recall that $\operatorname{dim} L_{x}^{j}=d$. Let $y, z \in W_{x, \varepsilon}^{j} \subset W, y \neq z$. Then the unit vector $\eta=$ $(z-y) /\|z-y\|$ is close to $V_{\left(x, \omega_{n}\right)}^{i_{j}}$ for every $n$. Therefore $\eta$ is close to $L_{x}^{j}$ and does not belong to the orthogonal complement $L_{x}^{j \perp}$ of $L_{x}^{j}$. Let $P_{y}$ denote the $(m-d)$-plane parallel to $L_{x}^{j \perp}$ and passing through $y \in U, P_{x}=L_{x}^{j \perp}$. For a tangent vector $w \in L_{x}^{j}$ at $x$ let $P_{x, w}$ be the $(m-d+1)$-plane $\left\{t w+z: t \in \mathbb{R}, z \in P_{x}\right\}$. If $U$ is small enough, then for any $y \in P_{x, w} \cap U$ the intersection $L_{y}^{j} \cap P_{x, w}$ is one-dimensional and there is a unique vector $v_{w}(y) \in L_{y}^{j} \cap P_{x, w}$ whose projection onto the $t$-axis in $P_{x, w}$ is $w$. Clearly $v_{w}(\cdot)$ is a continuous vector field on $U \cap P_{x, w}$ which depends continuously on $w$. Consider now the differential equation $\dot{y}=v_{w}(y)$ in $U \cap P_{x, w}$. We claim that for a small enough $U$ there is a unique integral curve $y=g_{w}(t)$ passing through $x$. To see that, note that any such curve is tangent to $L^{j}$ and therefore lies in $W$. Suppose there are two different integral curves $y=g_{i}(t), i=1,2, g_{i}(0)=0, g_{1}\left(t_{0}\right) \neq g_{2}\left(t_{0}\right)$. Let $\pi$ denote the orthogonal projection onto $L_{x}^{j}$. Then $\pi\left(g_{1}(t)\right)=\pi\left(g_{2}(t)\right)=t w$; i.e. $g_{1}(t)-g_{2}(t) \in L_{x}^{j \perp}$, which is a contradiction. 
Since $v_{w}$ depends continuously on $w$, the uniqueness of $g_{w}$ implies that it depends continuously on $w$. If $w_{1}$ and $w_{2}$ are not collinear, then the integral curves $g_{w_{1}}$ and $g_{w_{2}}$ lie in different planes and hence $g_{w_{1}}(t) \neq g_{w_{2}}(t)$ for any $t>0$. Therefore, if $U$ is small enough, then the restriction $\psi$ of the map $w \rightarrow g_{w}(1)$ to $L_{x}^{j} \cap U$ is a homeomorphism. Since no two points from $W_{x, \varepsilon}^{j}$ have the same projection on $L_{x}^{j}$, for a small $\varepsilon$ and properly chosen $U$ the image of $\psi$ is $W_{x, \varepsilon}^{j}$ and the plane $L_{x}^{j}$ is clearly tangent to the image at $x$. Let $y \in W_{x, \tilde{\varepsilon}}^{j}, \tilde{\varepsilon}<\varepsilon$. Then the above argument shows that for $\delta<\varepsilon-\tilde{\varepsilon}$ the set $W_{y, \delta}^{j}$ is a $C^{0}$-submanifold that coincides locally near $y$ with $W_{x, \varepsilon}^{j}$ and is tangent to $L_{y}^{j}$ at $y$. It follows that $W_{x, \varepsilon}^{j}$ is a $d$-dimensional $C^{1}$. submanifold tangent to $L^{j}$.

Let $W_{x}^{j}$ denote the set of points lying on piecewise $C^{1}$-curves passing through $x$ and tangent to $L^{j}$. It follows from above that each set $W_{x}^{j}$ is a complete submanifold of $A_{q}$ which depends continuously on $x$ in the $C^{1}$-topology. Clearly the sets $W_{x}^{j}$ partition $A_{q}$. Since $L^{j}$ is invariant under any diffeomorphism $f$ from the support of measure $\mu$, we have that $f\left(W_{x}^{j}\right)=W_{f x}^{j}$, and hence the foliation $W^{j}$ with leaves $W_{x}^{j}$ is also invariant under any such diffeomorphism. This finishes the proof of integrability of $L^{j}$.

Let $x \in A_{q}$. Then the set $\left\{\omega: L_{x}^{j} \subseteq V_{(x, \omega)}^{i_{j}}\right\}=\Omega_{x}$ has full $\nu$-measure by Corollary 3.10. If $\omega \in \Omega_{x}$ and $\varepsilon(x, \omega)=\varepsilon(\omega)$ is small enough, then, by Lemma 4.5 , the set $W_{x, \varepsilon(\omega)}^{j}$ is contained in the local stable manifold $W_{(x, \omega)}^{\xi}$. Hence, by Theorem 4.1

$$
\limsup _{n \rightarrow \infty} \frac{1}{n} \ln \operatorname{diam}\left(F^{n}(\omega) W_{x, \varepsilon(\omega)}^{j}\right) \leq \lambda_{j} .
$$

This finishes the proof of Theorem 4.2.

Since $\lambda_{j}<0$ and therefore, by Corollary 3.4 , any vector tangent to $L^{j}$ is exponentially contracted by $D F^{n}(\omega)$ with probability 1 , one may wonder whether the size $\varepsilon(x, \omega)$ of the 'stable' manifold $W_{x, \varepsilon(\omega)}^{j}$ can be chosen independently of $\omega$ and whether the global integral manifolds $W_{x}^{j}$ are exponentially contracted by $F^{n}(\omega)$ with probability 1. An obvious sufficient condition for this to be true is given in Remark 4.6. However, as Example 4.7 shows, in general the answer to both questions is negative.

4.6. Remark. Let $\lambda_{j}<0$ and assume that

$$
\lim _{n \rightarrow \infty} \frac{1}{n} \ln \left\|D F^{n}(\omega) \mid L^{j}\right\| \leq \lambda \leq 0 \quad \nu \text {-a.e. }
$$

where $\lambda<\lambda_{j+1}$. Then for a.e. $\omega$ all vectors are uniformly contracted by $D F^{n}(\omega)$ and hence any global integral manifold $W_{x}^{j}$ is exponentially contracted by $F^{n}(\omega)$ with asymptotic rate $\lambda$.

4.7. Example. Let $f$ be a diffeomorphism of the circle $S^{1}$ which we view as the interval $[0,1]$ with the ends identified. Then $1=$ length $(f([0,1]))=\int_{0}^{1}\left|f^{\prime}(x)\right| d x$. By the Jensen inequality,

$$
0=\ln \int_{0}^{1}\left|f^{\prime}(x)\right| d x \geq \int_{0}^{1} \ln \left|f^{\prime}(x)\right| d x,
$$

with equality if and only if $\left|f^{\prime}\right| \equiv$ const. Assume now that $f$ does not preserve the 
Lebesgue measure, i.e. $\left|f^{\prime}\right|$ is not constant. Then

$$
\int_{0}^{1} \ln \left|f^{\prime}(x)\right| d x<0
$$

Let $\theta_{n}, n=1,2, \ldots$, be a sequence of independent random variables uniformly distributed on $[0,1]$ and consider the sequence of independent random diffeomorphisms $F_{n} x=f(x)+\theta_{n}(\bmod 1), n=1,2, \ldots$ Then for each $x$ the transition probability $P(x, \cdot)$ of the Markov chain $X_{n}=F_{n} X_{n-1}$ is the Lebesgue measure $m$, and hence $m$ is invariant under $X_{n}$. Set $F^{n}=F_{n} \circ \ldots \circ F_{1}$ and let $\chi=\chi(x, \omega)$ denote the characteristic exponent. Since $m$ is obviously ergodic, the ergodic theorem implies that

$$
\begin{aligned}
\chi(x, \omega) & =\lim _{n \rightarrow \infty} \frac{1}{n} \ln \left|\left(F^{n}(\omega)\right)^{\prime}(x)\right|=\lim _{n \rightarrow \infty} \frac{1}{n} \sum_{k=1}^{n} \ln \left|F_{k}^{\prime}\left(F^{k-1} x\right)\right| \\
& =\int_{0}^{1} \ln \left|f^{\prime}(y)\right| d y
\end{aligned}
$$

for a.e. $\omega$. The last integral is negative by (4.19), and hence $\chi<0$. It follows from Theorem 4.2 that for any $x \in S^{1}$ and a.e. sequence of diffeomorphisms $\omega$ there is a neighbourhood $W_{x, \varepsilon}$ of size $\varepsilon=\varepsilon(x, \omega)$ that is exponentially contracted by $F^{n}(\omega)$. Suppose that $\varepsilon(x, \omega) \geq \varepsilon_{0}>0$ for some $x$ and $\nu$-a.e. $\omega$. Then $\varepsilon\left(F_{1} x, \theta \omega\right) \geq \alpha \varepsilon_{0}$ for a.e. $\omega$, where $\alpha=\min \left|f^{\prime}\right|$. Therefore, by the definition of $F_{1}, \varepsilon(x, \omega) \geq \alpha \varepsilon_{0}$ for a.e. $(x, \omega)$. Hence there exist finitely many points $x_{1}, \ldots, x_{k}$ such that their $\alpha \varepsilon_{0}$ neighbourhoods cover $S^{1}$ and $\varepsilon\left(x_{i}, \omega\right) \geq \alpha \varepsilon_{0}$ for $\nu$-a.e. $\omega$. It follows that there is an $\omega$ such that $F^{n}(\omega)$ contracts the whole circle exponentially. This is a contradiction. Hence the size of the local stable manifold is really random in this case.

4.8. Remark. Recall that stochastic flows generated by stochastic differential equations can be represented as compositions of independent identically distributed random diffeomorphisms. In this case the integrability condition (3.6), which was used in the proofs of Theorems 3.7 and 4.2, follows from Sobolev's embedding theorems provided the coefficients of stochastic differential equations are smooth enough. Moreover, even if the coefficients are not smooth enough, the main auxiliary statement, Corollary 5.3, can be obtained by using Gronwall's inequality and arguments similar to Totoki's proof of the multidimensional-time generalization of Kolmogorov's criteria for the path continuity of stochastic processes (see [E, p. 302]).

\section{Appendix: Hölder continuity of invariant subbundles}

5.1. Definition. Let $\Lambda$ be a (not necessarily complete) metric space, $H$ be a (separable, complete) Hilbert space and $\left\{E_{x}\right\}, x \in \Lambda$, be a family of subspaces, $E_{x} \subset H$. The family $\left\{E_{x}\right\}$ is called Hölder continuous in $x$ with exponent $\alpha, 0<\alpha \leq 1$, and constant $L, L>0$, if for any $x, y \in \Lambda$

$$
\operatorname{dist}\left(E_{x}, E_{y}\right) \stackrel{\text { def }}{=} \sup _{\substack{v \in E_{x} \\\|v\|=1}} \inf _{\substack{w \in E_{y} \\\|w\|=1}} \operatorname{dist}(v, w) \leq L \cdot(\operatorname{dist}(x, y))^{\alpha} .
$$

Let $X$ be a metric space, $\operatorname{diam}(X) \leq 1, H$ be a Hilbert space and $\left\{T_{i}(x)\right\}, i=$ $0,1, \ldots, x \in X$, be a sequence of families of bounded linear operators $T_{i}(x): H \rightarrow H$. 
Set

$$
T^{n}(x)=T_{n}(x) \circ \cdots \circ T_{1}(x) .
$$

5.2. ThEOREM. For $C>1$ and $\lambda<\mu$ let $\Lambda_{C, \lambda, \mu} \subset X$ be the (maybe empty) set of points $x$ for which there exists a splitting

$$
H=E_{x}^{\lambda} \oplus E_{x}^{\lambda \perp}
$$

such that for any positive integer $n$

$$
\begin{aligned}
& \left\|T^{n}(x) v\right\| \leq C e^{\lambda n}\|v\| \quad \text { if } v \in E_{x}^{\lambda}, \\
& \left\|T^{n}(x) w\right\| \geq C^{-1} e^{\mu n}\|w\| \quad \text { if } w \in E_{x}^{\lambda \perp} .
\end{aligned}
$$

Suppose there is $a>\max (\lambda, 0)$ and $0<\beta \leq 1$ such that

$$
\left\|T^{n}(x)-T^{n}(y)\right\| \leq e^{a n}(\operatorname{dist}(x, y))^{\beta}
$$

for any positive integer $n$ and any $x, y \in X$.

Then the family $\left\{E_{x}^{\lambda}\right\}$ is Hölder continuous in $x$ on $\Lambda_{C, \lambda, \mu}$ with exponent $((\lambda-\mu) /(\lambda-a)) \beta$ and constant $3 C^{2} e^{\mu-\lambda}$.

Proof. Set

$$
K_{x}^{n}=\left\{v \in H:\left\|T^{n}(x) v\right\| \leq 2 C e^{\lambda n}\|v\|\right\} .
$$

Let $v \in K_{x}^{n}, v=v^{\lambda}+v^{\perp}$, where $v^{\lambda} \in E_{x}^{\lambda}, v^{\perp} \in E_{x}^{\lambda \perp}$. By the triangle inequality $\left\|T^{n}(x) v\right\|=\left\|T^{n}(x)\left(v^{\lambda}+v^{\perp}\right)\right\| \geq\left\|T^{n}(x) v^{\perp}\right\|-\left\|T^{n}(x) v^{\lambda}\right\| \geq C^{-1} e^{\mu n}\left\|v^{\perp}\right\|-C e^{\lambda n}\|v\|$.

Therefore

and

$$
\left\|v^{\perp}\right\| \leq C e^{-\mu n}\left(\left\|T^{n}(x) v\right\|+C e^{\lambda n}\|v\|\right) \leq 3 C^{2} e^{(\lambda-\mu) n}\|v\|
$$

$$
\operatorname{dist}\left(v, E_{x}^{\lambda}\right) \leq 3 C^{2} e^{(\lambda-\mu) n}\|v\|
$$

Fix $a_{1}>\max (a, \lambda)$ and set

$$
\gamma=\left(\lambda-a_{1}\right) / \beta
$$

Let $x, y \in \Lambda_{C, \lambda, \mu}$. Since $\gamma<0$, there is a unique non-negative integer $n=n(x, y)$ such that

$$
e^{\gamma(n+1)}<\operatorname{dist}(x, y) \leq e^{\gamma n}
$$

For any $w \in E_{y}^{\lambda}$

$$
\begin{aligned}
\left\|T^{n}(x) w\right\| & \leq\left\|T^{n}(y) w\right\|+\left\|T^{n}(x)-T^{n}(y)\right\|\|w\| \\
& \leq C e^{\lambda n}\|w\|+e^{a n}(\operatorname{dist}(x, y))^{\beta}\|w\| \\
& \leq\left(C e^{\lambda n}+e^{a n} e^{\beta \gamma n}\right)\|w\| \leq 2 C e^{\lambda n}\|w\| .
\end{aligned}
$$

Hence $w \in K_{x}^{n}$ and $E_{y}^{\lambda} \subset K_{x}^{n}$. By symmetry, $E_{x}^{\lambda} \subset K_{y}^{n}$. It follows from (5.1) and (5.2) that

$$
\begin{aligned}
\operatorname{dist}\left(E_{x}^{\lambda}, E_{y}^{\lambda}\right) & \leq 3 C^{2} e^{(\lambda-\mu) n} \leq 3 C^{2} e^{\mu-\lambda}(\operatorname{dist}(x, y))(\lambda-\mu) / \gamma \\
& =3 C^{2} e^{\mu-\lambda}(\operatorname{dist}(x, y))^{\left((\lambda-\mu) /\left(\lambda-a_{1}\right)\right) \cdot \beta} .
\end{aligned}
$$

5.3. COROLlARY. Let $M$ be a Riemannian manifold with injectivity radius $r>0$ and let $\left\{f_{i}\right\}, i=1,2, \ldots$, be a sequence of differentiable maps $f_{i}: M \rightarrow M$ such that the differentials $D f_{i}$ satisfy

$$
\prod_{i=1}^{n}\left\|D f_{i}\right\|_{a} \leq C_{1} e^{a n}, \quad n=1,2, \ldots
$$

where $a, C_{1}>0,0<\sigma \leq 1$. Set $f^{n}=f_{n} \circ f_{n-1} \circ \cdots \circ f_{1}$. Fix $C>0$ and $\lambda<\mu$ and let 
$\Lambda_{C, \lambda, \mu}$ be the (maybe empty) set of points $x$ for which there exists a splitting

$$
T_{x} M=E_{x}^{\lambda} \oplus E_{x}^{\lambda 1}
$$

such that for any positive integer $n$

$$
\begin{aligned}
\left\|D_{x} f^{n} v\right\| & \leq C e^{\lambda n}\|v\| \quad \text { if } v \in E_{x}^{\lambda}, \\
\left\|D_{x} f^{n} w\right\| & \geq C^{-1} e^{\mu n}\|w\| \quad \text { if } w \in E_{x}^{\lambda \perp} .
\end{aligned}
$$

Then the family $\left\{E_{x}^{\lambda}\right\}$ is Hölder continuous in $x$ on $\Lambda_{C, \lambda, \mu}$ with constant $3 C^{2} e^{\mu-\lambda}$ and exponent $\alpha=((\lambda-\mu) /(\lambda-b)) \sigma$, where $b=\ln \left(2 C_{1}^{2}\right)+2 a-\sigma \ln r+|\lambda|$.

Proof. We will need the following lemma.

5.4. LeMma. For $n \geq 1$

$$
\operatorname{dist}\left(D_{x} f^{n}, D_{y} f^{n}\right) \leq e^{b n}(\operatorname{dist}(x, y))^{\sigma} \text {. }
$$

Proof. Assume that dist $\left(f^{n} x, f^{n} y\right) \geq r$ for some $n \geq 0$. Then, by (3.5),

$$
r \leq \operatorname{dist}\left(f^{n} x, f^{n} y\right) \leq \prod_{i=1}^{n}\left\|D f_{i}\right\|_{\sigma} \cdot \operatorname{dist}(x, y)
$$

and hence, by (5.3), dist $(x, y) \geq r C_{1}^{-1} e^{-a n}$. Therefore, by (3.4), (3.5), (5.3) and by the choice of $b$, for any $m \geq n$ we have

$$
\operatorname{dist}\left(D_{x} f^{m}, D_{y} f^{m}\right) \leq 2 \prod_{i=1}^{m}\left\|D f_{i}\right\|_{\sigma} \leq 2 C_{1} e^{a m} \leq e^{m b}(\operatorname{dist}(x \cdot y))^{\sigma},
$$

where we used the inequality dist $\left(D_{x} f^{m}, D_{y} f^{m}\right) \leq\left\|D_{x} f^{m}\right\|+\left\|D_{y} f^{m}\right\|$.

Hence it suffices to prove (5.4) when $\operatorname{dist}\left(f^{i} x, f^{i} y\right)<r$ for all $i=0,1, \ldots, n$. Note that (5.4) follows from (5.3) and the inequality

$$
\operatorname{dist}\left(D_{x} f^{n}, D_{y} f^{n}\right) \leq\left(\prod_{i=1}^{n}\left\|D f_{i}\right\|_{\sigma}\right)^{2} \cdot(\operatorname{dist}(x, y))^{\sigma}
$$

which we now prove by induction. For $n=1$ (5.5) follows from (3.5). Assume now that (5.5) holds true for $n=k$. For $n=k+1$ using (3.4), (3.5) and the inductive assumption we have:

$$
\begin{aligned}
\operatorname{dist}( & \left.D_{x} f^{k+1}, D_{y} f^{k+1}\right) \\
= & \left\|D_{x} f^{k+1}-P\left(f^{k+1} y, f^{k+1} x\right) \circ P(x, y)\right\| \\
= & \| D_{f^{k}} f_{k+1} \circ D_{x} f^{k} \\
& -P\left(f^{k+1} y, f^{k+1} x\right) D_{f^{k} y} f_{k+1} \circ P\left(f^{k} x, f^{k} y\right) \circ P\left(f^{k} y, f^{k} x\right) \circ D_{y} f^{k} \circ P(x, y) \| \\
\leq & \left\|D_{f^{k}} f_{k+1} \circ D_{x} f^{k}-P\left(f^{k+1} y, f^{k+1} x\right) \circ D_{f^{k} y} f_{k+1} \circ P\left(f^{k} x, f^{k} y\right) \circ D_{x} f^{k}\right\| \\
& +\| P\left(f^{k+1} y, f^{k+1} x\right) \circ D_{f^{k} y} f_{k+1} \circ P\left(f^{k} x, f^{k} y\right) \circ D_{x} f^{k} \\
& -P\left(f^{k+1} y, f^{k+1} x\right) \circ D_{f^{k}} f_{k+1} \circ P\left(f^{k} x, f^{k} y\right) \circ P\left(f^{k} y, f^{k} x\right) \circ D_{y} f^{k} \circ P(x, y) \| \\
\leq & \operatorname{dist}\left(D_{f^{k} x} f_{k+1}, D_{f^{k}} f_{k+1}\right) \cdot\left\|D_{x} f^{k}\right\|+\left\|D_{f^{k}} f_{n+1}\right\| \cdot \operatorname{dist}\left(D_{x} f^{k}, D_{y} f^{k}\right) \\
\leq & \left(\left\|D f_{k+1}\right\|_{\sigma}-\sup _{z}\left\|D_{z} f_{k+1}\right\|\right) \cdot\left(\operatorname{dist}\left(f^{k} x, f^{k} y\right)\right)^{\sigma} \cdot\left\|D_{x} f^{k}\right\| \\
& +\sup _{z}\left\|D_{z} f_{k+1}\right\| \cdot\left(\prod_{i=1}^{k}\left\|D f_{i}\right\|_{\sigma}\right)^{2} \cdot(\operatorname{dist}(x, y))^{\sigma} \\
\leq & \left(\prod_{i=1}^{k+1}\left\|D f_{i}\right\|_{\sigma}\right)^{2} \cdot(\operatorname{dist}(x, y))^{\sigma}
\end{aligned}
$$


since

$$
\operatorname{dist}\left(f^{k} x, f^{k} y\right) \leq \prod_{i=1}^{k}\left\|D f_{i}\right\|_{\sigma}
$$

Fix any points $x, y \in \Lambda_{C, \lambda, \mu}$ and set

$$
T_{i}(x)=D_{x} f^{i}, \quad T_{i}(y)=P\left(f^{i} y, f^{i} x\right) \circ D_{y} f^{i} \circ P(x, y) .
$$

Identify the tangent spaces $T_{f^{i}} M$ by any sequence of isometries with $H=T_{x} M$ and consider the discrete set $X=\bigcup_{n=0}^{\infty}\left(f^{n} x \cup f^{n} y\right)$ with the following distance function: $\operatorname{dist}^{\prime}\left(f^{i} x, f^{i} y\right)=\operatorname{dist}\left(f^{i} x, f^{i} y\right)$ if this distance is not greater than $r$, and the distance is $r$ in all other cases. By Theorem 5.2 and Lemma 5.4,

$$
\operatorname{dist}\left(E_{x}^{\lambda}, E_{y}^{\lambda}\right) \leq 3 C^{2} e^{\mu-\lambda}(\operatorname{dist}(x, y))^{\alpha} \text {. }
$$

\section{REFERENCES}

[BN] M. Brin \& Z. Nitecki. Absolute continuity of stable foliations in Hilbert space. Preprint, 1987.

[C] A. P. Carverhill. A nonrandom Lyapunov spectrum for non-linear stochastic dynamical systems. Stochastic 17 (1986), 253-287.

[D] J. L. Doob. Stochastic Processes. Wiley, New York, 1953.

[E] D. Elworthy. Stochastic Differential Equations on Manifolds. London Math. Soc. Lecture Notes, Cambridge Univ. Press, 1982.

[FHY] A. Fathi, M. Herman \& J. C. Yoccoz. Proof of Pesin's stable manifold theorem. In Geometric Dynamics (ed. J. Palis, Jr.), Lecture Notes in Math. 1007 (Springer, 1983), 177-215.

[HS] E. Hewitt \& K. Stromberg. Real and Abstract Analysis. Springer, New York, 1965.

[Ki] Yu. Kifer. Ergodic Theory of Random Transformations. Birkhauser, 1986.

[Ku ] H. Kunita. Stochastic differential equations and stochastic flows of diffeomorphisms. Lecture Notes in Math. 1097 (Springer, 1984), 143-303.

[L] F. Ledrappier. Quelques propriétés des exposants charactéristiques. Lecture Notes in Math. 1097 (Springer, 1984), 305-396.

[Or] S. Orey. Limit Theorems for Markov Chain Transition Probabilities. Van Nostrand Reinhold Co., London, 1971.

[Os] V. I. Oseledeč. A multiplicative ergodic theorem. Lyapunov characteristic numbers for dynamical systems. Trans. Moscow Math. Soc. 19 (1968), 197-231.

[P] Ya. B. Pesin. Families of invariant manifolds corresponding to non-zero characteristic exponents. Math. USSR Izvestija 10 (1976), 1261-1305.

[Re] D. Revuz. Markov Chains. North-Holland Publishing Co., Amsterdam, 1975.

[Ro] M. Rosenblatt. Markov Processes. Structure and Asymptotic Behavior. Springer, Berlin, 1971.

[Ru] D. Ruelle. Ergodic theory of differentiable dynamical systems. Publ. Math. IHES 50 (1979), 275-306.

[Rul] D. Ruelle. Characteristic exponents and invariant manifolds in Hilbert space. Annals of Math. 115 (1982), 243-290. 